\title{
MAJOR-MERGER GALAXY PAIRS IN THE COSMOS FIELD—MASS-DEPENDENT MERGER RATE EVOLUTION SINCE $z=1$
}

\author{
C. Kevin Xu ${ }^{1}$, Yinghe ZhaO ${ }^{2}$, N. Scoville ${ }^{3}$, P. CaPaK $^{4}$, N. Drory ${ }^{5,6}$, and Y. GaO ${ }^{2}$ \\ ${ }^{1}$ Infrared Processing and Analysis Center, California Institute of Technology 100-22, Pasadena, CA 91125, USA \\ ${ }_{2}^{2}$ Purple Mountain Observatory, Chinese Academy of Sciences, 2 West Beijing Road, Nanjing 210008, China \\ ${ }^{3}$ California Institute of Technology, MC 105-24, 1200 East California Boulevard, Pasadena, CA 91125, USA \\ ${ }^{4}$ Spitzer Science Center, California Institute of Technology, Mail Stop 220-6, Pasadena, CA 91125, USA \\ ${ }^{5}$ Instituto de Astronomía, Universidad Nacional Autónoma de México, A.P. 70-264, 04510 México, D.F., Mexico \\ ${ }^{6}$ Max-Planck Institut für extraterrestrische Physik, Giessenbachstrasse, 85748 Garching, Germany \\ Received 2011 September 7; accepted 2011 November 22; published 2012 February 16
}

\begin{abstract}
We present results of a statistical study of the cosmic evolution of the mass-dependent major-merger rate since $z=1$. A stellar mass limited sample of close major-merger pairs (the CPAIR sample) was selected from the archive of the COSMOS survey. Pair fractions at different redshifts derived using the CPAIR sample and a local $K$-bandselected pair sample show no significant variations with stellar mass. The pair fraction exhibits moderately strong cosmic evolution, with the best-fitting function of $f_{\text {pair }}=10^{-1.88( \pm 0.03)}(1+z)^{2.2( \pm 0.2)}$. The best-fitting function for the merger rate is $R_{\mathrm{mg}}\left(\mathrm{Gyr}^{-1}\right)=0.053 \times\left(M_{\mathrm{star}} / 10^{10.7} M_{\odot}\right)^{0.3}(1+z)^{2.2} /(1+z / 8)$. This rate implies that galaxies of $M_{\text {star }} \sim 10^{10}-10^{11.5} M_{\odot}$ have undergone $\sim 0.5-1.5$ major mergers since $z=1$. Our results show that, for massive galaxies $\left(M_{\text {star }} \geqslant 10^{10.5} M_{\odot}\right)$ at $z \leqslant 1$, major mergers involving star-forming galaxies (i.e., wet and mixed mergers) can account for the formation of both ellipticals and red quiescent galaxies (RQGs). On the other hand, major mergers cannot be responsible for the formation of most low mass ellipticals and RQGs of $M_{\text {star }} \lesssim 10^{10.3} M_{\odot}$. Our quantitative estimates indicate that major mergers have significant impact on the stellar mass assembly of the most massive galaxies $\left(M_{\text {star }} \geqslant 10^{11.3} M_{\odot}\right)$, but for less massive galaxies the stellar mass assembly is dominated by the star formation. Comparison with the mass-dependent (ultra)luminous infrared galaxies ((U)LIRG) rates suggests that the frequency of major-merger events is comparable to or higher than that of (U)LIRGs.
\end{abstract}

Key words: galaxies: evolution - galaxies: general - galaxies: interactions - galaxies: starburst

Online-only material: color figures

\section{INTRODUCTION}

Galaxy mergers have fascinated astronomers for a long time, ever since they were recognized (see the review of Schweizer 1996). Major mergers of galaxies of nearly equal mass stand out because of the more spectacular tidal and dynamical effects (Toomre 1978), and many nearby major mergers have been extensively studied (Toomre \& Toomre 1972; Whitmore \& Schweizer 1995; Hibbard \& van Gorkom 1996; Hibbard \& Yun 1999; Xu et al. 2000; Wang et al. 2004). It has been well documented that major mergers can induce enhanced star formation (Kennicutt et al. 1987; Xu \& Sulentic 1991), trigger extreme starbursts and active galactic nuclear (AGN) activities (Sanders et al. 1988; Sanders \& Mirabel 1996; Dasyra et al. 2006), and transform spiral galaxies into elliptical galaxies (Toomre 1978; Schweizer 1982; Genzel et al. 2001). They dominate among the extreme starbursts such as luminous infrared galaxies (LIRGs, with star formation rate $(\mathrm{SFR}) \gtrsim 20 M_{\odot} \mathrm{yr}^{-1}$ ) and ultra-luminous infrared galaxies (ULIRGs, with SFR $\gtrsim 200 M_{\odot} \mathrm{yr}^{-1}$; Sanders \& Mirabel 1996). On the other hand, statistically, major mergers play minor roles in processes such as star formation and mass growth of $z \sim 0$ galaxies in general. Only $\sim 1 \%-2 \%$ of galaxies are involved in close major mergers (Xu et al. 2004; Patton \& Atfield 2008; Domingue et al. 2009), and only 2\%-3\% of star formation rate density (SFRD) in the $z=0$ universe is due to close major mergers (Xu et al. 2010).

Are mergers more important in the earlier universe? Indeed, in the hierarchical structure formation paradigm of the contemporary cosmology, galaxy and dark matter halo $(\mathrm{DMH})$ merging is one of the most significant processes affecting the evolution of structures in the early universe, and is largely responsible for the growth of massive DMHs and the buildup of galaxies (Kauffmann et al. 1993; Lacey \& Cole 1993; Khochfar \& Burkert 2005). Many observations of intermediate/high-redshift peculiar galaxies and galaxy pairs have found strong evolution in the merger rate, up to $(1+z)^{3-6}$ (Brinchmann et al. 1998; LeFévre et al. 2000; Conselice et al. 2003, 2009; Conselice 2006; Kampczyk et al. 2007; Kartaltepe et al. 2007; Rawat et al. 2008), and show evidence of mergers dominating the total star formation rate in the universe of $z \gtrsim 1$ (Zheng et al. 2004; Hammer et al. 2005; Bridge et al. 2007). On the other hand, weak merger rate evolution $\left(\sim(1+z)^{0.5}\right)$ has been found by other studies of intermediate/high-redshift mergers (Carlberg et al. 2000; Lin et al. 2004; Lotz et al. 2008; Robaina et al. 2010; Man et al. 2011), and many authors have argued that at $z \gtrsim 1$ the SFRD is still predominantly contributed by isolated late-type galaxies rather than by mergers (Flores et al. 1999; Bell et al. 2005; Lotz et al. 2008; Jogee et al. 2009). Furthermore, mergers may no longer be the predominant population among LIRGs and even ULIRGs at $z \gtrsim 1$ (Melbourne et al. 2005; Daddi et al. 2010).

In this paper, we address the controversy on the merger rate evolution using data in the COSMOS field (Scoville et al. 2007). Major reasons for the controversy include different definitions of major mergers, errors in the merger timescale, and biases in the merger sample selections (see Xu et al. 2010; Hopkins et al. 2010b). Studies using merger samples selected from peculiar galaxies (LeFévre et al. 2000; Conselice et al. 2003, 2009; Conselice 2006; Jogee et al. 2009) are vulnerable to contaminations due to minor mergers and to uncertainties of the timescale for detectable peculiarities such 
as tidal tails, bridges, plumes, and other distortions (Lotz et al. 2010). In contrast, we selected our merger samples from close $\left(5 h^{-1} \mathrm{kpc} \leqslant r_{\text {proj }} \leqslant 20 h^{-1} \mathrm{kpc}\right.$ ) major-merger pairs (stellar mass ratios $\leqslant 2.5)$. These pairs have reasonably well understood merger timescales (Kitzbichler \& White 2008; Lotz et al. 2010).

Our pair sample is selected from the photo- $z$ catalog of the COSMOS field (Ilbert et al. 2009) and is stellar mass limited, including massive galaxies $\left(M_{\text {star }} \geqslant 10^{9} M_{\odot}\right)$ in the photo$z$ range of $0.2 \leqslant z_{\text {phot }} \leqslant 1$. The COSMOS sample has the best photo- $z$ 's, measured using data of $\sim 30$ photometric bands covering the entire UV-infrared range, for more than 100,000 galaxies with nearly $100 \%$ completeness (Ilbert et al. 2009). This enables us to obtain a pair sample that is $\sim 70 \%$ complete. By comparison, the pair samples in the studies of Patton et al. (2002), Lin et al. (2004), and Bundy et al. (2009), using pairs selected from spectroscopic surveys, are only 10\%-20\% complete. Given the rather complex spectroscopic selection functions in those studies, the corrections for the incompleteness may lead to substantial uncertainties in the results.

Photo- $z$ selected pairs of $0.2 \leqslant z_{\text {phot }} \leqslant 1.2$ in the COSMOS field were studied by Kartaltepe et al. (2007, hereafter K07). Their sample is different from ours in two major respects: (1) it is not confined to major mergers (i.e., no constraint on the mass ratios or luminosity ratios between primaries and secondaries), and (2) it is an absolute magnitude limited sample $\left(M_{V} \leqslant-19.8\right)$. With more rigorously defined major-merger pair samples, our study shall improve upon the results of K07. Also, with well-determined stellar mass for every galaxy in the sample, we shall study the mass dependence of the merger rate evolution.

We will confine our analysis to galaxy pairs of $z \leqslant 1$, because the photo- $z$ 's and stellar mass estimates of $z>1$ galaxies are less accurate (Ilbert et al. 2010). We will derive the merger rates for galaxies of different stellar masses and redshifts, and separate dry mergers (E+E pairs) and wet/mixed mergers $(S+S$ and $S+E$ mergers). The selection of COSMOS pairs in the redshift range of $0.2 \leqslant z \leqslant 1$ is described in Section 2. Corrections for the incompleteness and for contaminations by spurious pairs are presented in Section 3. A $z=0$ pair sample, which sets the local benchmark for the evolution study, is presented in Section 4. The mass-dependent merger rate and its evolution since $z=1$ are presented in Section 5. The contributions of major mergers to the galaxy assembly and elliptical galaxy formation since $z=1$ are investigated in Section 6, and comparisons to the mass and redshift dependence of the (U)LIRGs' abundance are in Section 7. Section 8 is devoted to a summary of our main results. Throughout this paper, we adopt the $\Lambda$-cosmology with $\Omega_{\mathrm{m}}=0.3$ and $\Omega_{\Lambda}=0.7$, and $H_{0}=70\left(\mathrm{~km} \mathrm{~s}^{-1} \mathrm{Mpc}^{-1}\right)$.

\section{THE COSMOS PAIR (CPAIR) SAMPLE}

We selected major-merger pair candidates using a parent sample of galaxies constructed from that used by Drory et al. (2009, hereafter D09) in their study of galaxy stellar mass function (GSMF), which was in turn selected from the COSMOS photo- $z$ catalog (Ilbert et al. 2009) using the following criteria: $0.2 \leqslant z \leqslant 1, K_{s}<24$, and $i_{\mathrm{AB}}^{+}<25.5$.

The D09 sample has 138,001 galaxies, divided into four photo- $z$ bins with widths of $\Delta z=0.2$, and into "active" (star-forming galaxies (SFGs)) and "passive" (red quiescent galaxies (RQGs)) populations according to the spectral energy distribution (SED) type of the best-fitting template (Ilbert et al. 2009). The stellar mass of galaxies, $M_{\text {star }}$, is derived through a stellar population synthesis model fitting (the Chabrier initial
Table 1

Parent Sample

\begin{tabular}{lcccccc}
\hline \hline$z_{\min }$ & $z_{\max }$ & $\begin{array}{c}\text { Volume } \\
\left(10^{6} \mathrm{Mpc}^{3}\right)\end{array}$ & $\begin{array}{c}\log \left(M_{\min }\right) \\
\left(M_{\odot}\right)\end{array}$ & \multicolumn{2}{c}{ Number of Galaxies $^{\mathrm{a}}$} \\
\cline { 5 - 7 } & & 0.56 & 9.0 & 6787 & 2039 & 8826 \\
\hline 0.2 & 0.4 & 1.23 & 9.4 & 6169 & 1526 & 7695 \\
0.4 & 0.6 & 1.92 & 9.8 & 6745 & 1981 & 8726 \\
0.6 & 0.8 & 2.53 & 10.2 & 6610 & 2287 & 8897 \\
0.8 & 1.0 & & & & & \\
\hline
\end{tabular}

Notes.

${ }^{\text {a }}$ Number of galaxies with $\log \left(M_{\text {star }}\right) \geqslant \log \left(M_{\min }\right)$.

b SFGs ("active galaxies") and RQGs ("passive galaxies") classifications were taken from D09.

mass function (IMF)), using the photo- $z$ and photometric data in the $u^{*}$ (Canada-France-Hawaii Telescope (CFHT)), $B_{J}, V_{J}, g^{+}$, $r^{+}, i^{+}, z^{+}$(Subaru), $J$ (UKIRT), and $K_{s}$ (CFHT) bands. Typical uncertainties in $M_{\text {star }}$ are between 0.1 dex and 0.3 dex at $68 \%$ confidence level, depending on spectral type and the signal-tonoise ratio of the photometry (D09). In the four photo- $z$ bins, the completeness limits for SFGs and RQGs are $\log \left(M_{\text {star }} / M_{\odot}\right)=$ $[8.3,8.9,9.2,9.4]$ and $\log \left(M_{\text {star }} / M_{\odot}\right)=[8.9,9.2,9.8,10.1]$, respectively.

For galaxies in our parent sample, the stellar mass is taken from D09. In order to be complete for both SFGs and RQGs, we imposed a mass $\operatorname{limit}\left(\log \left(M_{\min }\right)\right)$ on each of the photo- $z$ bins in the parent sample. The mass limit and the number of galaxies above the limit are listed in Table 1 . There are 34,144 galaxies in the parent sample.

The pair sample is also divided into four photo- $z$ bins. The selection criteria are as follows.

1. The primary galaxy has $\log \left(M_{\text {star }}\right) \geqslant \log \left(M_{\text {lim }}\right)$, with $\log \left(M_{\lim } / M_{\odot}\right)=[9.4,9.8,10.2,10.6]$ for the four redshift bins, respectively. $M_{\text {lim }}$ 's are 0.4 dex above the $M_{\min }$ 's of the parent sample (Table 1).

2. The difference in $M_{\text {star }}$ between the primary galaxy and the secondary galaxy is less than 0.4 dex: $\Delta \log \left(M_{\text {star }}\right) \leqslant 0.4$.

3. The redshift difference between the two components, $\Delta z_{\text {phot }}=\left|z_{\text {phot }}^{\text {pri }}-z_{\text {phot }}^{2 \text { nd }}\right|$, satisfies $\Delta z_{\text {phot }} /\left(1+z_{\text {phot }}^{\text {pri }}\right) \leqslant 0.03$.

4. The projected physical separation $\left(r_{\text {proj }}\right)$ is in the range of $5 h^{-1} \mathrm{kpc} \leqslant r_{\text {proj }} \leqslant 20 h^{-1} \mathrm{kpc}$.

Compared to the selection criteria for local pairs described in $\mathrm{Xu}$ et al. (2004), we replaced the rest-frame $K$-band selection by a stellar mass selection in criteria (1) and (2). The Spitzer Infrared Array Camera (IRAC) $3.6 \mu \mathrm{m}$ and $4.5 \mu \mathrm{m}$ bands, which encompass the rest-frame $K$-band emission for galaxies of $0.6 \lesssim z \leqslant 1$, have relatively low angular resolution compared to the Hubble Space Telescope (HST)/ground-based optical and NIR data. Using IRAC data would have resulted in larger confusion errors in the stellar mass for pairs with separation $\lesssim 2^{\prime \prime}$. At the same time, it was shown in D09 that for field galaxies of $z \leqslant 1$ the stellar mass derived using the $H S T$ /ground-based optical and NIR data is nearly identical to that derived using data including the IRAC fluxes (Ilbert et al. 2010).

Criteria (1) and (2) guarantee that our pair sample is not affected by the "missing secondary" bias (Xu et al. 2010). Criterion (3) is set to minimize the contamination of spurious pairs while ensuring that the completeness of the sample is not significantly compromised by the photo- $z$ error. This issue will be addressed in detail in Section 3.

Using these criteria, 417 pair candidates were selected. In order to exclude spurious pairs due to imaging artifacts, visual inspections were carried out on the HST Advanced Camera for 
Table 2

Sample of Paired Galaxies in COSMOS

\begin{tabular}{|c|c|c|c|c|c|c|c|c|c|c|}
\hline \multirow[t]{2}{*}{$z_{\min }$} & \multirow[t]{2}{*}{$z_{\max }$} & \multicolumn{3}{|c|}{ Number of Galaxies ${ }^{\mathrm{a}}$} & \multirow{2}{*}{$\begin{array}{c}\log \left(M_{\lim }\right) \\
\left(M_{\odot}\right)\end{array}$} & \multicolumn{5}{|c|}{ Number of Galaxies with $M_{\text {star }} \geqslant M_{\text {lim }}$ b } \\
\hline & & $\begin{array}{c}\text { In Iso. } \\
\text { Pairs }\end{array}$ & $\begin{array}{l}\text { In Multi. } \\
\text { Systems }\end{array}$ & Total & & $\begin{array}{c}\text { In Iso. } \\
\text { Pairs }\end{array}$ & $\begin{array}{l}\text { In Multi. } \\
\text { Systems }\end{array}$ & Total & SFGs & RQGs \\
\hline 0.2 & 0.4 & 144 & 6 & 150 & 9.4 & 128 & 5 & 133 & 78 & 55 \\
\hline 0.4 & 0.6 & 100 & 3 & 103 & 9.8 & 93 & 3 & 96 & 61 & 35 \\
\hline 0.6 & 0.8 & 144 & 22 & 166 & 10.2 & 126 & 20 & 146 & 109 & 37 \\
\hline 0.8 & 1.0 & 174 & 24 & 198 & 10.6 & 131 & 21 & 152 & 81 & 71 \\
\hline
\end{tabular}

Notes.

${ }^{a}$ Including all primaries and all secondaries.

${ }^{\mathrm{b}}$ Including all primaries and those secondaries with $\log \left(M_{\text {star }}\right) \geqslant \log \left(M_{\lim }\right)$.

Surveys (ACS) images (F814 band; Koekemoer et al. 2007). Among 417 pair candidates, 335 were covered by the $H S T$ survey. All pair candidates outside the area of HST-ACS imaging $(82 / 417=19.7 \%)$ were dropped from the final pair sample. Fourteen spurious pairs were identified: eight have wrong astrometry (i.e., no source appears at the sky coordinates) for at least one of the two galaxies and six are pieces of single large disk galaxies.

Some galaxies were found repetitively in multiple pair candidates. These 40 pair candidates consist of 17 triplets and 1 quartet, including 55 galaxies. The remaining 281 candidates are isolated pairs, including 562 galaxies. Because galaxies in triplets/quartets are less than $10 \%$ of the total sample, we will not distinguish them from paired galaxies. Our final COSMOS Pair sample (hereafter CPAIR) includes 617 paired galaxies, found in both isolated pairs and multiple systems. Among them, 527 (sum of the bold numbers in Table 2, including both primaries and secondaries) have $\log \left(M_{\text {star }}\right) \geqslant \log \left(M_{\text {lim }}\right)$, and the remaining (90, all being secondaries) have $\left(\log \left(M_{\lim }\right)-0.4\right) \leqslant \log \left(M_{\text {star }}\right)<\log \left(M_{\lim }\right)$. Statistics of the sample are listed in Table 2.

\section{CPAIR SAMPLE: INCOMPLETENESS AND SPURIOUS PAIRS FRACTION}

Much of the discrepancies between different results on merger rate evolution can be attributed to various biases causing incompleteness (missing of true mergers) and contaminations of spurious mergers in merger samples. Therefore, it is important to investigate thoroughly all such biases and correct them in merger statistics.

\subsection{Incompleteness due to Missing Very Close Pairs}

Photometric data of the photo- $z$ catalog (Ilbert et al. 2009) were obtained using the SExtractor in dual mode (Bertin \& Arnouts 1996). Images in all bands were degraded to a common point-spread function (PSF) of FWHM $=1$ 1..5, and the photometry was done with a constant aperture of $r=1$.'.5 (Capak et al. 2007). Because of the limited angular resolution of the photo- $z$ catalog, very close pairs with angular separation $\epsilon \lesssim 2^{\prime \prime}$ are incomplete in the pair sample. Exploiting the COSMOS HST-ACS lensing catalog (Leauthaud et al. 2007, 2010), we estimated this incompleteness to be $[0.01,0.06,0.08,0.20]$ for the four redshift bins, with no significant mass dependence. The full analysis can be found in Appendix A.

\subsection{Incompleteness due to Photo-z Errors and Spurious Pairs due to Projection}

For pairs of $\epsilon>2$ ". 0 , a major cause of the incompleteness is due to photo- $z$ errors, which have a non-negligible probability

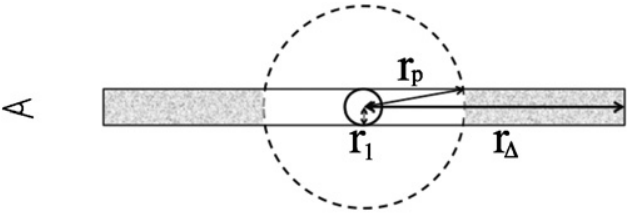

Figure 1. Illustration for Equation (1). $r_{1}$ is the maximum projected separation in the pair selection, $r_{p}$ is the outer boundary of physical (i.e., gravitationally bound) pairs, and $r_{\Delta}$ is the distance range along the line of sight that corresponds to the pair selection criterion for the photo- $z$ difference: $\left|\Delta z_{\text {phot }}\right| /\left(1+z_{\text {phot }}\right) \leqslant$ 0.03 . The shadowed areas are places where spurious companions are located.

of being so large that a real pair with $\Delta v<500 \mathrm{~km} \mathrm{~s}^{-1}$ (corresponding to $\Delta z /(1+z)<0.0017)$ can have a measured $\Delta z_{\text {phot }} /\left(1+z_{\text {phot }}\right)>0.03$ and therefore be missed by the CPAIR sample. Also, the photo- $z$ selection criterion and the photo- $z$ errors can introduce spurious pairs whose velocity difference $\Delta v$ is larger than $500 \mathrm{~km} \mathrm{~s}^{-1}$. Using Monte Carlo simulations, we estimated the incompleteness and the spurious pair fraction (hereafter SPF) to be $[0.21,0.21,0.23,0.25]$ and $[0.07,0.08$, $0.10,0.09]$, respectively, for the four redshift bins. The full analysis is presented in Appendix B.

\subsection{Clustering Effect on Spurious Pair Contaminations}

Bell et al. (2006) found that in the COMBO-17 survey, the projected two-point correlation functions of massive galaxies with $0.4<z_{\text {phot }} \leqslant 0.8$ can be well fitted by a power-law $w(r) \propto r^{-\gamma}$ down to $r=15 \mathrm{kpc}$, with the value of the powerindex $\gamma$ consistent with 2. Based on this result, we made a simple estimation for the effect of galaxy clustering that was neglected in the Monte Carlo simulations. It should be pointed out that, different from Patton et al. (2000) and Bell et al. (2006), we assumed that the boundary separating physical (i.e., gravitationally bound) and unphysical pairs, $r_{p}$, is much larger than $r_{1}=20 h^{-1} \mathrm{kpc}$, the separation limit in our pair selection. This is because the merger timescales derived by Lotz et al. (2010), as adopted in this work (Section 5.2), are for close pairs with projected separation $r \leqslant 20 h^{-1} \mathrm{kpc}$.

For a given two-point correlation function $\xi(r)=\left(r_{0} / r\right)^{\gamma}$, the additional $\operatorname{SPF}(\eta)$ due to clustering can be estimated as follows:

$$
\eta=\frac{4 \pi n \int_{r_{p}}^{r_{\Delta}}\left(r_{0} / r\right)^{\gamma} r^{2} d r \int_{0}^{\arcsin \left(r_{1} / r\right)} \sin (\theta) d \theta}{4 \pi n\left[\int_{0}^{r_{1}}\left(r_{0} / r\right)^{\gamma} r^{2} d r+\int_{r_{1}}^{r_{\Delta}}\left(r_{0} / r\right)^{\gamma} r^{2} d r \int_{0}^{\arcsin \left(r_{1} / r\right)} \sin (\theta) d \theta\right]} .
$$

The numerator on the right-hand side of the equation is the probability of finding spurious companions near a galaxy in both foreground and background (in the shadowed areas in Figure 1). 
Table 3

Completeness and Reliability Corrections for CPAIR Sample

\begin{tabular}{|c|c|c|c|c|c|c|c|c|}
\hline \multirow[t]{2}{*}{$z_{\min }$} & \multirow[t]{2}{*}{$z_{\max }$} & \multicolumn{3}{|c|}{ Completeness Correction } & \multicolumn{4}{|c|}{ Reliability Correction } \\
\hline & & $\begin{array}{l}\text { Due to Missing } \\
\text { Very Close Pairs }\end{array}$ & $\begin{array}{c}\text { Due to Photo- } z \\
\text { Errors }\end{array}$ & Combined & $\begin{array}{c}\text { Due to Random } \\
\text { Projection }\end{array}$ & $\begin{array}{c}\text { Due to } \\
\text { Clustering }\end{array}$ & $\begin{array}{c}\text { Due to Pairs of } \\
\Delta v>500 \mathrm{~km} \mathrm{~s}^{-1}\end{array}$ & Combined \\
\hline 0.2 & 0.4 & $0.99 \pm 0.01$ & $0.79 \pm 0.05$ & $0.78 \pm 0.05$ & $0.93 \pm 0.03$ & $0.94 \pm 0.05$ & $0.91 \pm 0.03$ & $0.80 \pm 0.06$ \\
\hline 0.4 & 0.6 & $0.94 \pm 0.03$ & $0.79 \pm 0.05$ & $0.74 \pm 0.05$ & $0.92 \pm 0.03$ & $0.94 \pm 0.05$ & $0.91 \pm 0.03$ & $0.79 \pm 0.06$ \\
\hline 0.6 & 0.8 & $0.92 \pm 0.03$ & $0.77 \pm 0.05$ & $0.71 \pm 0.05$ & $0.90 \pm 0.03$ & $0.94 \pm 0.05$ & $0.91 \pm 0.03$ & $0.77 \pm 0.06$ \\
\hline 0.8 & 1.0 & $0.80 \pm 0.04$ & $0.75 \pm 0.05$ & $0.60 \pm 0.05$ & $0.91 \pm 0.03$ & $0.94 \pm 0.05$ & $0.91 \pm 0.03$ & $0.78 \pm 0.06$ \\
\hline
\end{tabular}

Here, $r_{\Delta}$ is $\sim 100 \mathrm{Mpc}$, corresponding to the pair selection criterion of $\left|\Delta z_{\text {phot }}\right| /\left(1+z_{\text {phot }}\right) \leqslant 0.03$. The denominator is the probability of finding both real and spurious companions with a projected separation of $r \leqslant r_{1}$. The relations between parameters $r_{1}, r_{p}$, and $r_{\Delta}$ are illustrated in Figure 1. For $\gamma=2$ and $r_{1} \ll r_{p} \ll r_{\Delta}$, Equation (1) can be approximated by

$$
\eta=\frac{r_{1} / r_{p}}{\pi}
$$

Assuming $r_{p}=100 h^{-1} \mathrm{kpc}$, Equation (2) gives $\eta=0.06$. It is worth noting that (1) $\eta$ is comparable to the SPF obtained by the Monte Carlo simulations for the random associations; (2) $\eta$ is constant against the redshift; (3) given the uncertainties in $r_{p}$ and $\gamma$, we shall assume a relatively large error of 0.05 for $\eta$; and (4) this correction also applies to pairs selected spectroscopically because the condition $r_{\Delta} \gg r_{p}$ is still valid even for the selection criterion of $\Delta v \leqslant 500 \mathrm{~km} \mathrm{~s}^{-1}$.

\subsection{Fraction of Physical Pairs with $\Delta v>500 \mathrm{~km} \mathrm{~s}^{-1}$}

We excluded physical pairs with $500 \mathrm{~km} \mathrm{~s}^{-1}<\Delta v \lesssim$ $1000 \mathrm{~km} \mathrm{~s}^{-1}$ from the merger rate analysis since they have very uncertain and long merger timescales. These high $\Delta v$ pairs, found in the group/cluster environments (Domingue et al. 2009), are not included in the above estimates for projected unphysical pairs. Here we make a separate correction for them. Using local pairs taken from Domingue et al. (2009), selected using nearly identical selection criteria as the CPAIR sample (cf. Section 4) except that spec- $z$ were used and they include all pairs (isolated or in groups/clusters) with $\Delta v_{\text {spec }}<1000 \mathrm{~km} \mathrm{~s}^{-1}$, we find that the fraction of physical pairs with $\Delta v>500 \mathrm{~km} \mathrm{~s}^{-1}$ is $9.6 \% \pm 3.0 \%$ (Appendix C).

\subsection{Completeness and Reliability of the CPAIR Sample}

In Table 3, we listed estimates of the correction factors for subsamples in the four redshift bins. The combined completeness correction factor is the product of that due to missing very close pairs and that due to photo- $z$ errors. The combined reliability correction factor, defined as $1-$ SPF, is the product of that due to random projections, the additional correction due to the clustering effect, and that due to the contamination of physical pairs with $\Delta v>500 \mathrm{~km} \mathrm{~s}^{-1}$. The combined completeness correction factor varies in the range of $0.60-0.78$ between the four redshift bins. The combined reliability correction factor $(\sim 0.79)$ is rather constant against the redshift.

As an independent check, exploiting a sample of spec- $z$ pairs (the ZPAIR sample) selected from the zCOSMOS survey (Lilly et al. 2007), we empirically analyzed the completeness and reliability of the CPAIR sample. This resulted in an estimate of $0.86 \pm 0.12$ for the completeness correction factor due to photo$z$ errors (the incompleteness due to missing very close pairs cannot be checked with spec- $z$ pairs), which is consistent (within $1 \sigma$ ) with the result of the Monte Carlo simulations (Table 3). The estimate for the reliability correction is also $0.86 \pm 0.12$, again consistent (within $1 \sigma$ ) with the values of the combined reliability correction in Table 3 . The details of the analysis are presented in Appendix D.

\section{LOCAL PAIR SAMPLE}

Pair statistics in the local universe were carried out using an updated version of the KPAIR sample by Domingue et al. (2009), a close major-merger pair sample selected in the $K$ band from cross matches between the Two Micron All Sky Survey (2MASS) and the Sloan Digital Sky Survey (SDSS)DR5 galaxies. The update includes following modifications.

1. Stellar masses of galaxies in KPAIR and in its parent sample are multiplied by a factor of $10^{-0.39}$. This is the average difference between the mass estimated using the total $K_{s}$-band luminosity and a Salpeter IMF (Domingue et al. 2009), and the mass estimated using a Kroupa IMF by Kauffmann et al. (2003). Because the mass estimated using the Kroupa IMF and that using the Chabrier IMF are nearly identical (Kauffmann et al. 2003), this modification makes the masses in the local KPAIR sample and those in the CPAIR sample consistent.

2. In order to avoid possible bias due to the local overdensity (associated with the local super-cluster), a lower redshift cutoff of $v \geqslant 2000 \mathrm{~km} \mathrm{~s}^{-1}(z \geqslant 0.0067)$ is introduced.

3. Pairs with $500 \mathrm{~km} \mathrm{~s}^{-1}<\Delta v \leqslant 1000 \mathrm{~km} \mathrm{~s}^{-1}$ are excluded. Most of these pairs are in cluster environment. Excluding them improves the accuracy of the merger rate estimate.

4. The magnitude limit is set at $K_{s}=12.5$, the completeness limit of the KPAIR sample.

There are 18,081 galaxies in the parent sample that are brighter than $K_{s}=12.5$, of which 14,813 have measured redshifts (redshift completeness of $B_{\mathrm{z} \text {-comp }}=0.82$ ), and 14,218 are in the range of $0.0067 \leqslant z \leqslant 0.1$. The new paired galaxies sample has 221 galaxies, all brighter than $K_{s}=12.5$. Among them 188 are in pairs with two measured redshifts of $\Delta v_{\text {spec }} \leqslant 500 \mathrm{~km} \mathrm{~s}^{-1}$. These redshifts are in the range of $0.0067 \leqslant z \leqslant 0.1$, with the median of $z=0.042$. The remaining 33 galaxies are in single-redshift pairs (i.e., only one of the component galaxies having measured redshift).

\section{MASS-DEPENDENT MERGER RATES}

\subsection{Pair Fraction}

The local pair fraction is calculated using the following formula:

$$
f_{\text {pair }, 0}=\frac{B_{\text {z-comp }} \times(1-\eta)}{A_{0}} \times \frac{N_{2 z}+N_{1 z} \times\left(1-Q_{\text {spurious }}\right)}{N_{G, 0}},
$$


where $B_{\mathrm{z} \text {-comp }}=0.82$ is the redshift completeness of the parent sample, $A_{0}=0.89$ is the completeness of the local pair sample (Domingue et al. 2009), and $(1-\eta)=0.94$ is the clustering related reliability correction factor found in Section 3.3. $N_{2 z}$ and $N_{1 z}$ are numbers of galaxies in pairs of two measured redshifts and single redshifts, respectively, and $N_{G, 0}$ is the number of galaxies in the parent sample with measured redshift (in the range of $0.0067 \leqslant z<0.1)$. $Q_{\text {spurious }}=0.2$ is the probability for a single-redshift pair to be a spurious pair (Domingue et al. 2009). Note that $A_{0} \neq B_{\mathrm{z} \text {-comp }}^{2}$ because pairs with single measured redshifts were included. Also, we supplemented the SDSS redshifts of paired galaxies with redshifts found in the literature and in our own redshift observations (Domingue et al. 2009).

The pair fractions in the COSMOS field are estimated as follows:

$$
f_{\text {pair }}=\frac{Q_{\text {reli }}}{C_{\text {comp }} \times\left(1-D_{\mathrm{ACS}}\right)} \times \frac{N_{\mathrm{pg}}}{N_{G}},
$$

where $Q_{\text {reli }}=[0.80,0.79,0.77,0.78]$ is the reliability and $C_{\text {comp }}=[0.78,0.74,0.71,0.60]$ is the completeness (for the four photo- $z$ bins) of the pair sample, respectively; $D_{\mathrm{ACS}}=$ 0.197 is the fraction of pair candidates without ACS images (not included in the final pair sample, see Section 2); $N_{\mathrm{pg}}$ is the number of interacting galaxies in the pair sample; and $N_{G}$ is the number of galaxies in the parent sample.

The relative error of the pair fraction (i.e., error $/ f_{\text {pair }}$ ) can be estimated as the quadratic sum of the random error $\sigma_{\text {rms }}$ and the cosmic variance $\sigma_{\text {vari }} .^{7}$

$$
\sigma^{2}=\sigma_{\mathrm{rms}}^{2}+\sigma_{\mathrm{vari}}^{2}
$$

The random (binomial statistics) error is

$$
\sigma_{\mathrm{rms}}^{2}=\frac{1-f_{\text {pair }}}{N_{\mathrm{pg}}}
$$

For the local sample, we adopted the approximation $N_{\mathrm{pg}}=$ $N_{2 z}+N_{1 z}\left(1-Q_{\text {spurious }}\right)$. The cosmic variance is given by (Peebles 1980; Somerville et al. 2004)

$$
\sigma_{\text {vari }}^{2}=J_{2}(\gamma) \times\left(r_{0} / r_{\text {samp }}\right)^{\gamma},
$$

where $r_{0}$ and $\gamma$ are the parameters in the two-point correlation function $\xi(r)=\left(r_{0} / r\right)^{\gamma}, r_{\text {samp }}$ is the radius of the sampling volume, and $J_{2}$ is a function of $\gamma$ :

$$
J_{2}=\frac{72}{(3-\gamma)(4-\gamma)(6-\gamma) 2^{\gamma}} .
$$

The correlation function parameters for local galaxies of different masses were taken from Zehavi et al. (2005). For galaxies of $z \geqslant 0.2$, we assumed $\gamma=1.8$ and derived the $r_{0}$ values by interpolating the measurements for galaxies of different masses and redshifts by Zehavi et al. (2005), Meneux et al. (2008), and Foucaud et al. (2010). Cosmic variances for the integral pair fractions (i.e., not divided into mass bins) were taken from that for galaxies in the mass bin of $10.6<\log \left(M_{\text {star }} / M_{\odot}\right) \leqslant 11$.

\footnotetext{
7 The pair fraction, $f_{\text {pair }}$, is proportional to the probability of finding a second galaxy within a spatial separation $r$ from a given galaxy:

$P(r)=4 \pi n \int_{0}^{r}[1+\xi(r)] r^{2} d r$, where $n$ is the number density of galaxies and $\xi$ is the two-point correlation function. Hence $f_{\text {pair }}$ is proportional to $n$, and therefore the cosmic variance has the same effect on $f_{\text {pair }}$ as on the number density.
}

The pair fractions so calculated are listed in Table 4 and plotted in Figure 2. For local galaxies, we confirm the conclusion of Domingue et al. (2009) that there is no significant mass dependence of the pair fraction. The integral pair fraction at $z=0$ is $1.3 \% \pm 0.1 \%$, slightly lower than the result of Domingue et al. (2009) which is $1.6 \% \pm 0.1 \%$. The difference is due to two factors: (1) the sample of Domingue et al. (2009) includes all pairs of $\Delta v \leqslant 1000 \mathrm{~km} \mathrm{~s}^{-1}$ whereas local pairs in this work are restricted to pairs of $\Delta v \leqslant 500 \mathrm{~km} \mathrm{~s}^{-1}$, and (2) the application of the clustering related reliability correction factor $((1-\eta)=0.94)$.

There is no evidence for a significant mass dependence of the pair fractions in higher photo- $z$ bins, either. The trend for pair fractions to increase with redshift can be seen in all mass bins, though with substantial scatter. The major reason for the large scatter is the cosmic variance, given the relatively small volume explored by the COSMOS survey in each photo- $z$ bin (Table 1). In particular, there is a strong density enhancement in the photo- $z$ bin of $0.2 \leqslant z<0.4$ (D09), which biases the pair fraction toward a higher value ( $f_{\text {pair }}$ is proportional to the density). Cosmic variance often dominates the total error in the pair fraction: its contribution is usually $>50 \%$ except in those bins where the number of paired galaxies, $N_{\mathrm{pg}}$, is less than 10 (hence the random error is large). In Figure 3, we plot the redshift dependence of the integral pair fraction (pair fraction of all galaxies regardless of the stellar mass), and that of the pair fraction for galaxies in the mass bin $10.6<\log \left(M_{\text {star }} / M_{\odot}\right) \leqslant 11$ (the bin encompassing the $M_{\text {star }}^{*}$ ). These two results are very close to each other, in agreement with our conclusion that the pair fraction does not vary significantly with stellar mass.

The least-squares fit to the redshift dependence of the integral pair fractions is $f_{\text {pair }}=10^{-1.88( \pm 0.03)}(1+z)^{2.2( \pm 0.2)}$. For the pair fractions in the mass bin of $10.6<\log \left(M_{\text {star }} / M_{\odot}\right) \leqslant 11$, the best fit is $f_{\text {pair }}=10^{-1.84( \pm 0.05)}(1+z)^{2.1( \pm 0.3)}$.

\subsection{Differential Major-merger Rate}

The differential major-merger rate is the probability of each galaxy to be involved in a major merger per Gyr: $R_{\mathrm{mg}} \propto$ $f_{\text {pair }} / T_{\mathrm{mg}}$, where $T_{\mathrm{mg}}$ is the merger timescale in Gyr. Because the physical process of a galaxy merger is very complex (see Hopkins et al. 2010a for a review), $T_{\mathrm{mg}}$ has been a major source of uncertainty in the merger rate studies. In the literature the most common approach has been the approximation of $T_{\mathrm{mg}}$ by the dynamical friction timescale (Binney \& Tremaine 1987; Patton et al. 2000; Jiang et al. 2008; Kitzbichler \& White 2008). Kitzbichler \& White (2008) studied the $T_{\mathrm{mg}}$ in a semi-analytical model built on the results of the Millennium Simulation. They assumed circular orbits to estimate the dynamical friction process and found relatively weak mass and redshift dependence in the form of $T_{\mathrm{mg}} \propto M_{\mathrm{star}}^{-0.3}(1+z / 8)$. However, their dynamical friction time is only appropriate for small satellite galaxies at large radii. For the massive close major-merger pairs in our samples, it becomes a very poor approximation because of two issues (Hopkins et al. 2010b). (1) Angular momentum loss at these radii is not dominated by the dynamical friction, but rather by exchange in strong resonances between the baryonic components that act much more efficiently. (2) By these radii, even the initially circular orbits have become highly radial, leading to shorter merger times.

Based on these reasons, we instead estimated $T_{\mathrm{mg}}$ using the results of Lotz et al. (2010). They carried out high-resolution hydrodynamical simulations for a large number of galaxy 
Table 4

Mass-dependent Pair Fraction

\begin{tabular}{|c|c|c|c|c|c|c|c|c|c|c|}
\hline \multirow[t]{2}{*}{ Mass Bin } & \multicolumn{2}{|c|}{$z=0$} & \multicolumn{2}{|c|}{$0.2 \leqslant z \leqslant 0.4$} & \multicolumn{2}{|c|}{$0.4<z \leqslant 0.6$} & \multicolumn{2}{|c|}{$0.6<z \leqslant 0.8$} & \multicolumn{2}{|c|}{$0.8<z \leqslant 1$} \\
\hline & $f_{\text {pair }}$ & $N_{\mathrm{pg}} / N_{G}$ & $f_{\text {pair }}$ & $N_{\mathrm{pg}} / N_{G}$ & $f_{\text {pair }}$ & $N_{\mathrm{pg}} / N_{G}$ & $f_{\text {pair }}$ & $N_{\mathrm{pg}} / N_{G}$ & $f_{\text {pair }}$ & $N_{\mathrm{pg}} / N_{G}$ \\
\hline $9.4 \leqslant \log (M) \leqslant 9.8$ & $0.014 \pm 0.011$ & $2 / 126$ & $0.027 \pm 0.006$ & $44 / 2034$ & $\cdots$ & $0 / 0$ & $\cdots$ & $0 / 0$ & $\cdots$ & $0 / 0$ \\
\hline $9.8<\log (M) \leqslant 10.2$ & $0.011 \pm 0.006$ & $7 / 524$ & $0.020 \pm 0.006$ & $24 / 1482$ & $0.030 \pm 0.006$ & 44/1977 & $\cdots$ & $0 / 0$ & $\cdots$ & $0 / 0$ \\
\hline $10.2<\log (M) \leqslant 10.6$ & $0.013 \pm 0.002$ & $41 / 2775$ & $0.043 \pm 0.010$ & $39 / 1174$ & $0.030 \pm 0.007$ & $34 / 1524$ & $0.038 \pm 0.007$ & $71 / 2542$ & $\cdots$ & $0 / 0$ \\
\hline $10.6<\log (M) \leqslant 11.0$ & $0.014 \pm 0.002$ & $97 / 5826$ & $0.035 \pm 0.011$ & $19 / 706$ & $0.025 \pm 0.007$ & $18 / 955$ & $0.044 \pm 0.008$ & $62 / 1913$ & $0.045 \pm 0.010$ & $110 / 3229$ \\
\hline $11.0<\log (M) \leqslant 11.4$ & $0.011 \pm 0.002$ & $68 / 4520$ & $0.042 \pm 0.020$ & $6 / 183$ & $(0)$ & $0 / 267$ & $0.021 \pm 0.008$ & $10 / 645$ & $0.061 \pm 0.015$ & $39 / 1051$ \\
\hline $11.4<\log (M) \leqslant 11.8$ & $0.014 \pm 0.007$ & $8 / 417$ & $(0)$ & $0 / 17$ & (0) & $0 / 29$ & $(0)$ & $0 / 65$ & $0.048 \pm 0.029$ & $3 / 103$ \\
\hline Total & $0.013 \pm 0.001$ & $223 / 14188$ & $0.030 \pm 0.010$ & $132 / 5596$ & $0.026 \pm 0.007$ & $96 / 4752$ & $0.038 \pm 0.009$ & $143 / 5165$ & $0.056 \pm 0.011$ & $152 / 4383$ \\
\hline
\end{tabular}




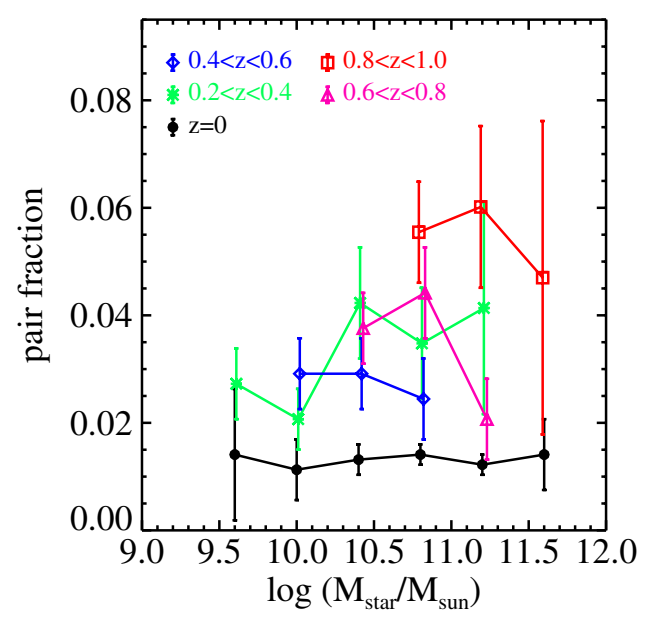

Figure 2. Mass-dependent pair fractions at different redshifts.

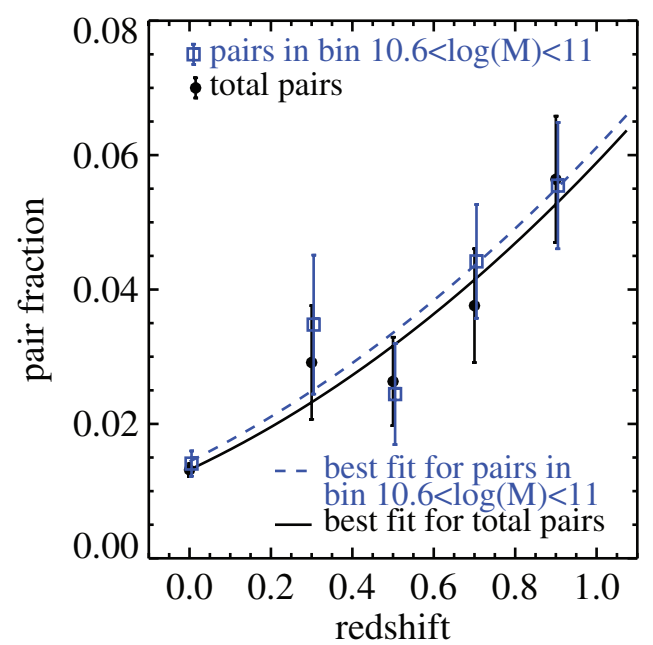

Figure 3. Pair fraction evolution. The solid line is the least-squares fit to total pair fraction (pair fraction of all galaxies regardless of the stellar mass) vs. redshift relation, specified by $f_{\text {pair }}=10^{-1.88( \pm 0.03)}(1+z)^{2.2( \pm 0.2)}$. The dashed line is the least-squares fit to the pair fractions in the mass bin of $10.6<\log \left(M_{\text {star }} / M_{\odot}\right) \leqslant 11$, specified by $f_{\text {pair }}=10^{-1.84( \pm 0.05)}(1+z)^{2.1( \pm 0.3)}$.

mergers with diverse initial conditions and derived $T_{\mathrm{mg}}$ at different projected separations in a view-angle averaged format. These simulated mergers have line-of-sight velocity difference $\Delta v \leqslant 500 \mathrm{~km} \mathrm{~s}^{-1}$, identical to the pairs in our sample. Nine mergers in Lotz et al. (2010) have baryonic mass ratios $\leqslant 3$ (i.e., one 1:1 merger and eight 3:1 mergers) with $M_{\text {star }} \simeq 10^{10.7} M_{\odot}$. In the bin of $5 h^{-1} \mathrm{kpc} \leqslant r_{\text {proj }} \leqslant 20 h^{-1} \mathrm{kpc}$, these nine mergers have an average merging timescale of $T_{\mathrm{mg}}=0.30 \pm 0.06 \mathrm{Gyr}$. The three 1:1 mergers in Lotz et al. (2010) have stellar masses in the range of $10^{9.7}-10^{10.7} M_{\odot}$, and their $T_{\mathrm{mg}}$ 's in the $5 h^{-1} \mathrm{kpc} \leqslant r_{\text {proj }} \leqslant 20 h^{-1} \mathrm{kpc}$ bin show a weak mass dependence of $T_{\mathrm{mg}} \sim M_{\mathrm{star}}^{-0.2}$, consistent with that found by Kitzbichler \& White (2008). Because Lotz et al. (2010) did not study the redshift dependence of $T_{\mathrm{mg}}$, and because their sample is too small (three mergers) to derive a meaningful mass dependence, we adopt the relation found by Kitzbichler \& White (2008), namely $T_{\mathrm{mg}} \propto M_{\text {star }}^{-0.3}(1+z / 8)$. These dependences are sufficiently weak that the associated uncertainties will not have any significant effect on our results. The final merger timescale

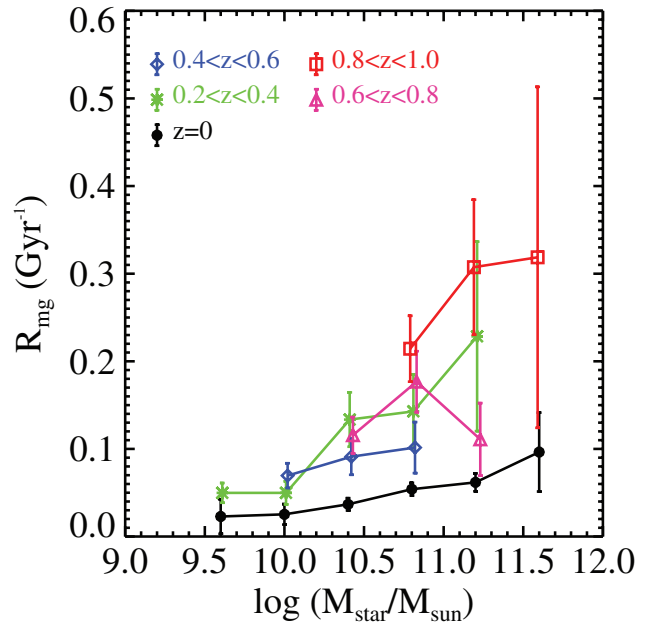

Figure 4. Mass-dependent differential merger rate in different redshifts.

we adopted is

$$
T_{\mathrm{mg}}=0.3 \mathrm{Gyr} \times\left(\frac{M_{\mathrm{star}}}{10^{10.7} M_{\odot}}\right)^{-0.3}\left(1+\frac{z}{8}\right) .
$$

And the differential merger rate for major mergers of mass ratio $\leqslant 3$ is

$$
R_{\mathrm{mg}}=A \times f_{\mathrm{pair}} / T_{\mathrm{mg}},
$$

where $A=1.19$ is the factor converting the pair fraction in this work (for mergers of mass ratio $\mu \leqslant 2.5$ ) to that of mergers of mass ratio $\mu \leqslant 3$. Here we assumed that $f_{\text {pair }} \propto \log \left(\mu_{\max }\right)$ (Appendix E). In Table 5 and Figure 4, we present our results on $R_{\mathrm{mg}}$.

Using the least-squares fit to the pair fraction evolution, $f_{\text {pair }}=10^{-1.88( \pm 0.03)}(1+z)^{2.2( \pm 0.2)}$, and Equation (9), we derived a best-fit function for the mass-dependent $R_{\mathrm{mg}}$ evolution:

$$
R_{\mathrm{mg}}\left(M_{\mathrm{star}}, z\right)=0.053 \times\left(\frac{M_{\mathrm{star}}}{10^{10.7} M_{\odot}}\right)^{0.3} \frac{(1+z)^{2.2}}{1+z / 8}\left(\mathrm{Gyr}^{-1}\right) .
$$

This is in very good agreement with the prediction of the highresolution $\Lambda$ CDM $N$-body simulation of Stewart et al. (2009): $d N / d t\left(\mathrm{Gyr}^{-1}\right)=0.06 \times\left[\left(1+L / L_{*}\right) / 2\right] \times(1+z)^{2.1}$. Integrating this merger rate over time, we find that the probability for individual galaxies to be involved in a major merger since $z=1$ is $0.8 \times\left(M_{\text {star }} / 10^{10.7} M_{\odot}\right)^{0.3}$. Accordingly, on average, massive galaxies of $M_{\text {star }} \sim 10^{10}-10^{11.5} M_{\odot}$ have undergone $\sim 0.5-1.5$ times mergers since $z=1$.

\subsection{Comparisons with Previous Results}

Our results on the mass independence of the local pair fraction (filled circles in Figure 2) are in agreement with those of Domingue et al. (2009) and Patton \& Atfield (2008) while contradicting the results of $\mathrm{Xu}$ et al. (2004), which were derived using a small sample of 19 pairs. For pairs of higher redshifts, Bundy et al. (2009) found a trend of positive mass dependence, which was not confirmed by our results. In Figure 5, our results are compared to those of Bundy et al. (2009). In order to compensate for the difference in the mass ratios in the two works $(\mu \leqslant 2.5$ in this work and $\mu \leqslant 4.0$ in Bundy et al. 2009), both results are converted to the $f_{\text {pair }}$ for $\mu \leqslant 3$ mergers. In Appendix $\mathrm{E}$, it is shown that 
Table 5

Mass-dependent Differential Merger Rate

\begin{tabular}{|c|c|c|c|c|c|}
\hline \multirow[t]{2}{*}{ Mass Bin } & \multicolumn{5}{|c|}{$R_{\mathrm{mg}}\left(\mathrm{Gyr}^{-1}\right)$} \\
\hline & $z=0$ & $0.2 \leqslant z \leqslant 0.4$ & $0.4<z \leqslant 0.6$ & $0.6<z \leqslant 0.8$ & $0.8<z \leqslant 1$ \\
\hline $9.4 \leqslant \log (M) \leqslant 9.8$ & $0.023 \pm 0.019$ & $0.050 \pm 0.011$ & $\cdots$ & $\cdots$ & $\cdots$ \\
\hline $9.8<\log (M) \leqslant 10.2$ & $0.025 \pm 0.012$ & $0.049 \pm 0.013$ & $0.068 \pm 0.014$ & $\cdots$ & $\cdots$ \\
\hline $10.2<\log (M) \leqslant 10.6$ & $0.037 \pm 0.008$ & $0.132 \pm 0.030$ & $0.089 \pm 0.020$ & $0.112 \pm 0.020$ & $\cdots$ \\
\hline $10.6<\log (M) \leqslant 11.0$ & $0.055 \pm 0.007$ & $0.140 \pm 0.042$ & $0.099 \pm 0.029$ & $0.171 \pm 0.033$ & $0.211 \pm 0.037$ \\
\hline $11.0<\log (M) \leqslant 11.4$ & $0.062 \pm 0.011$ & $0.226 \pm 0.107$ & $\cdots$ & $0.108 \pm 0.040$ & $0.302 \pm 0.076$ \\
\hline $11.4<\log (M) \leqslant 11.8$ & $0.096 \pm 0.046$ & $\cdots$ & $\cdots$ & $\cdots$ & $0.313 \pm 0.191$ \\
\hline
\end{tabular}

the pair fraction $f_{\text {pair }}$ increases proportionally with $\log \left(\mu_{\max }\right)$. Accordingly, the pair fractions from this work were scaled up by a factor of $1.19=\log (3) / 0.4$, and those of Bundy et al. (2009) were scaled down by a factor of $0.80=\log (3) / 0.6$. The results of Bundy et al. (2009) might have suffered from large uncertainties. Those obtained using their "method I" (projected pairs without any redshift information for the companions), which are plotted in Figure 5, were based on pair samples with high contaminations ( $\sim 60 \%-70 \%)$ of unphysical pairs; and those from their "method II" (spectroscopic and/or photometric redshifts for both components) were based on small samples (3-15 paired galaxies in each mass/redshift bin). de Ravel et al. (2009) claimed evidence for strong mass dependence of the evolutionary index of the pair fraction, in the sense that low mass pairs have strong pair fraction evolution $(m=3.13 \pm 1.54)$ and high mass pairs have weak evolution $(m=0.52 \pm 2.07)$. However, their results are very uncertain, as indicated by their large errors. de Ravel et al. (2009) also claimed evidence for strong evolution ( $m=4.73 \pm 2.01)$ in optically faint pairs $\left(M_{B} \leqslant-18-Q z, Q=1.11\right)$ and for weak evolution $(m=$ $1.50 \pm 0.76)$ in optically bright pairs $\left(M_{B} \leqslant-18.77-Q z\right)$. But the low evolutionary index of the bright pairs was obtained only when they included in their fit the $z=0$ pair fraction of de Propris et al. (2007), one of the highest local pair fractions in the literature (Figure 6). Indeed, when calculated in the same way as for the evolutionary index of faint pairs (i.e., fitting only high- $z$ data points), the index of bright pairs is $m=3.07 \pm 1.68$, consistent with that for faint pairs.

In Figure 6, our results on the cosmic evolution of the integral pair fraction are compared with those taken from the literature. It shows that the evolutionary rate derived from our results is in between those for the strong evolution (e.g., the result of K07) and for weak evolution (e.g., the result of Lin et al. 2008), respectively. Actually, our pair fractions in the photo- $z$ bins of $z=0.2-1.0$ agree well with those of $\mathrm{K} 07$ in the same redshift range. The marginally significant difference between the two evolutionary rates is mainly due to the relatively low pair fraction at $z=0$, which may be caused by an incompleteness associated with the "missing secondary" bias (Xu et al. 2004), and the relatively high pair fraction at $z=1.3$ in the results of $\mathrm{K} 07$. We clearly see much stronger evolution than that of Lin et al. (2008). Their results, based on spectroscopically confirmed pair samples in incomplete redshift surveys, may have relatively large statistical uncertainties because of the large correction factors (a factor of $>3$ ) for the incompleteness. The color-based pre-selection of their redshift surveys may also introduce biases in the pair selection, given the significant influence of galaxy-galaxy interaction on optical colors (Larson \& Tinsley 1978).

In the literature, pair fractions are often compared to merger fractions estimated using counts of peculiar galaxies (Conselice et al. 2003, 2009; Lotz et al. 2008; Jogee et al. 2009). In general, the latter are higher than the former, because (1) of contaminations from irregular galaxies (Jogee et al. 2009); (2) morphologically selected merger samples based on the $\mathrm{G}-\mathrm{M}_{20}$ method (Lotz et al. 2008, 2010) include minor mergers; and (3) the merger timescales for morphologically selected merger samples based on the CAS (Concentration, Asymmetry, and Clumpiness) method are longer than the merger timescales of close majormerger pairs (Conselice et al. 2009). Given the different merger timescales for close pairs and for peculiar galaxies, it is more appropriate to compare the differential merger rates $R_{\mathrm{mg}}$. In Figure 7, we compare the inverse of the $R_{\mathrm{mg}}, \Gamma=1 / R_{\mathrm{mg}}$ (Conselice et al. 2009), of morphologically selected mergers by Conselice et al. (2009) and by Lotz et al. (2008) with that of $M_{\text {star }}^{*}$ galaxies $\left(\log \left(M_{\text {star }}^{*} / M_{\odot}\right) \sim 10.8\right.$; Ilbert et al. 2010) in close major-merger pairs in this work and in Bundy et al. (2009). The $\Gamma$ parameter derived using paired galaxies in our sample (the inverse of Equation (11)) and that of Conselice et al. (2009) derived using morphologically selected mergers are in very good agreement. The higher $\Gamma$ values of Bundy et al. (2009) are likely due to the relatively long merging timescale they adopted from Kitzbichler $\&$ White (2008). On the other hand, the low $\Gamma$ values and lack of evolution of Lotz et al. (2008) are because of the inclusion of minor mergers in their sample. Our results are in good agreement with the prediction of the default (semi-empirical) model of Hopkins et al. (2010a).

\section{MAJOR MERGERS, ELLIPTICAL GALAXY FORMATION, AND GALAXY ASSEMBLY}

\subsection{Mass-dependent Volume Merger Rate}

The volume merger rate, $R_{V}$, measures the frequency of merger events in a given volume in the universe. Here we define the mass-dependent $R_{V}$ in terms of the stellar mass of the merger remnant, which is the total stellar mass of the two galaxies involved in the merging (ignoring the mass of stars formed during the merger):

$$
R_{V}\left(M_{\mathrm{star}}, z\right)=0.5 \times R_{\mathrm{mg}}\left(M_{\mathrm{star}} / 10^{0.2}, z\right) \phi\left(M_{\mathrm{star}} / 10^{0.2}, z\right),
$$

where $\phi(M, z)$ is the GSMF of galaxies in the parent sample (D09), and the factor of 0.5 is due to the fact that every major-merger event involves two galaxies of similar mass. We also assume that on average the mass of a merger remnant is 0.2 dex higher than that of individual galaxies involved in the merger. This is because, under the assumption that mass ratio distribution is flat (Appendix E), pairs in our sample have a mean mass ratio of 0.2 dex. Therefore, the logarithm of the mean ratio between the total mass of a pair and that of the primary is $\log \left(1+10^{-0.2}\right) \simeq$ 0.2 . Our results on the $R_{V}$ are presented in Table 6 . 


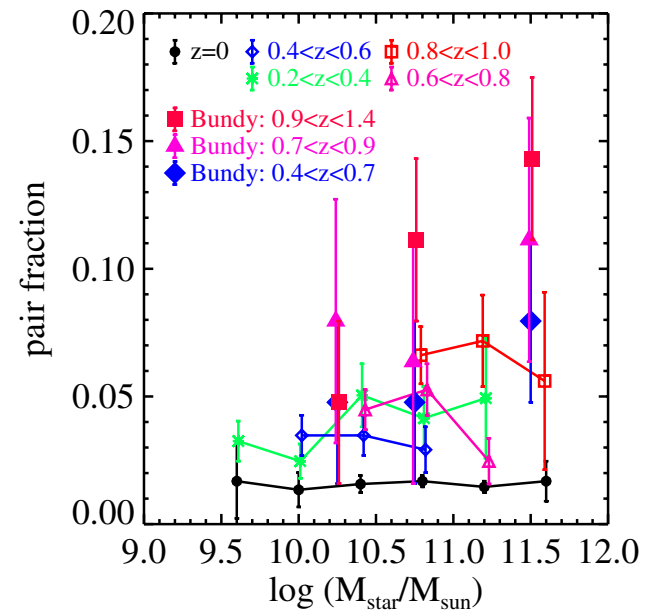

Figure 5. Comparisons of mass-dependent pair fractions of this work with those of Bundy et al. (2009). Both results are converted to the $f_{\text {pair }}$ for mergers of mass ratio, $\mu, \leqslant 3$ (see the text).

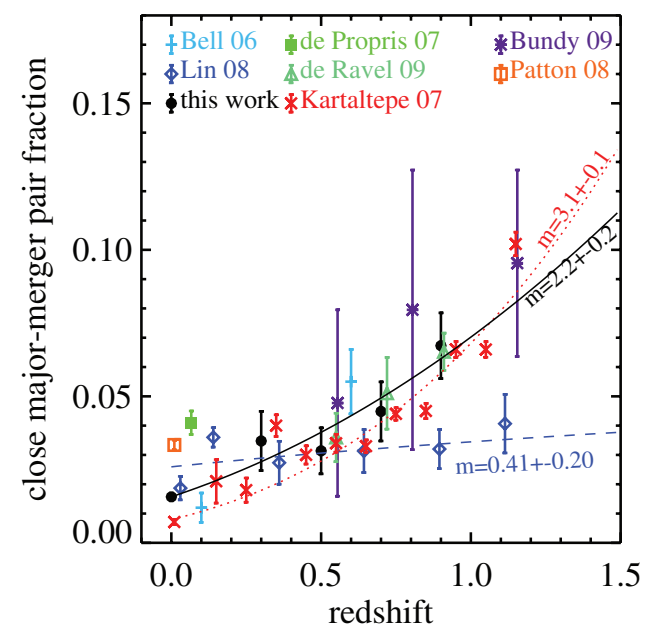

Figure 6. Comparisons of observed pair fractions in the literature. When it is appropriate, results of different authors were corrected so they are consistent with a common definition of close major-merger pairs with the maximum projected separation of $r_{\text {proj, } \max }=20 h^{-1} \mathrm{kpc}$ and the maximum primaryto-secondary mass ratio $\mu_{\max }=3$. Results of pair samples of different $r_{\text {proj,max }}$ were corrected by assuming $f_{\text {pair }} \propto\left(r_{\text {proj,max }}\right)^{3-\gamma}$ with $\gamma=2$ (Bell et al. 2006). For example, results of Lin et al. (2008) were divided by a factor of 1.5 because they had $r_{\text {proj,max }}=30 h^{-1} \mathrm{kpc}$. The results of Bell et al. (2006) had $r_{\text {proj, } \max }=30 \mathrm{kpc}$, corresponding to $r_{\text {proj, } \max }=21 h^{-1} \mathrm{kpc}$ for $h=0.7$, very close to the $r_{\text {proj,max }}=20 h^{-1} \mathrm{kpc}$ and therefore no correction was applied. Results of pair samples of different mass ratio limits were corrected by assuming $f_{\text {pair }} \propto \log \left(\mu_{\max }\right)$ (Figure 18). These include results of this work $\left(\log \left(\mu_{\max }\right)=0.4\right)$, Bundy et al. (2009) and de Ravel et al. (2009) (both having $\left.\log \left(\mu_{\max }\right)=0.6\right)$, and Patton \& Atfield (2008) $\left(\log \left(\mu_{\max }\right)=0.3\right)$. This correction was not applied to results from pair samples without mass ratio cutoff (e.g., K07; de Propris et al. 2007).

In Figure 8, we compare our results with the average growth rate of elliptical galaxies (dashed line) and that of RQGs (dotted line) between $z=0.3-0.9$, estimated using the differences between their GSMFs at $z=0.3$ and $z=0.9$, taken from Ilbert et al. (2010), divided by $3.88 \mathrm{Gyr}$ (the time span corresponding to the redshift interval of $[0.3,0.9])$. The solid line is the average volume merger rate calculated using Equation (12) by replacing $R_{\mathrm{mg}}$ with its best fit (Equation (11)) and averaged over the same redshift range of 0.3-0.9. It shows that major mergers can fully account for the formation of both massive ellipticals and RQGs $\left(M_{\text {star }} \geqslant 10^{10.5} M_{\odot}\right)$. This contradicts Bundy et al. (2009) who

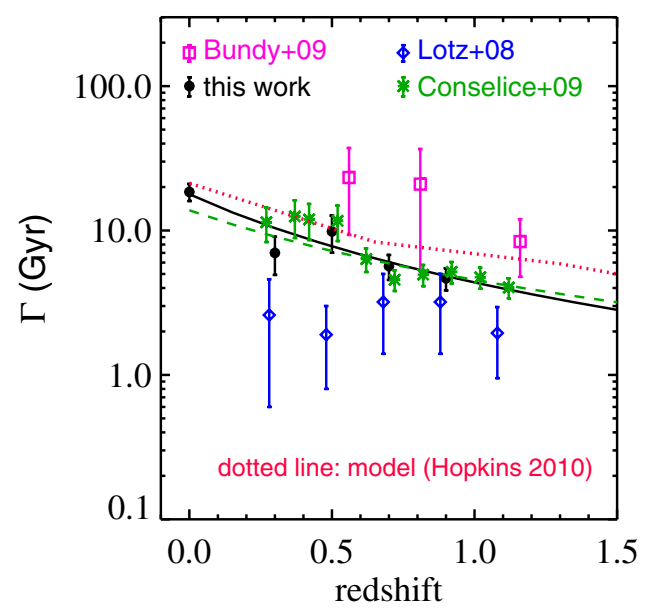

Figure 7. Redshift dependence of $\Gamma=1 / R_{\mathrm{mg}}$. The values for morphologically selected mergers obtained by Conselice et al. (2009) and of Lotz et al. (2008) were taken from Figure 7 of Conselice et al. (2009). $R_{\mathrm{mg}}$ 's of Bundy et al. (2009) were scaled down by a factor of $0.80=\log (3) / 0.6$ to make it consistent with the merger rate for $\mu \leqslant 3$ mergers. The solid line is the inverse of Equation (11) of this work. The dashed line is the best fit of Conselice et al. (2009). The dotted line is the model prediction of Hopkins et al. (2010a).

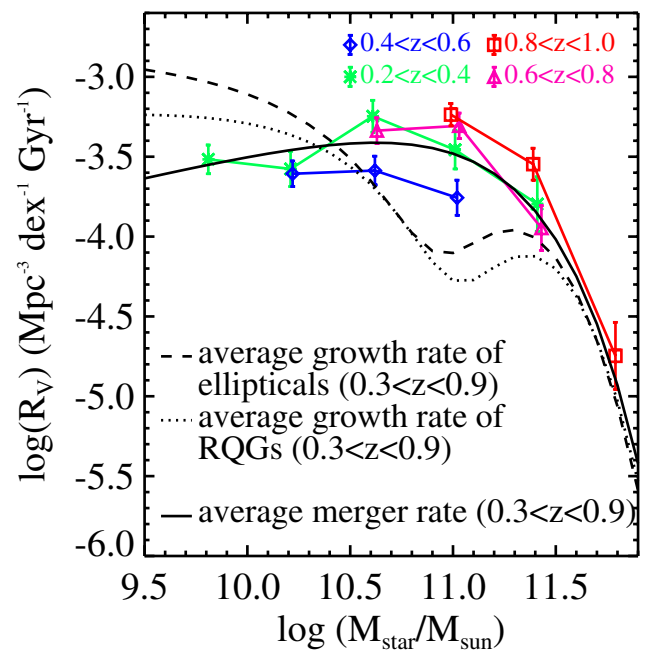

Figure 8. Data points with error bars are mass-dependent volume merger rates in different redshift bins. The dashed line is the average growth rate of ellipticals between $z=0.3-0.9$ and dotted line that of RQGs, estimated using the mass function of Ilbert et al. (2010). The solid line is the average volume merger rate calculated using Equation (12) by replacing $R_{\mathrm{mg}}$ with its best fit (Equation (11)) and averaged over the same redshift range of 0.3-0.9.

concluded that the major-merger rate is too low to fully explain the formation of RQGs since $z=1$. The major reason for the contradiction is due to the difference in the adopted merger timescales in this work and in Bundy et al. (2009). Our $T_{\mathrm{mg}}$, derived from the results of Lotz et al. (2010), is about a factor of two shorter than that used by Bundy et al. (2009). There is also a difference in the formation rates of ellipticals and RQGs adopted in this work (estimated from results of Ilbert et al. 2010) and in Bundy et al. (2009). The latter is about 50\%-100\% higher than the former.

We define "dry mergers" ("wet mergers") as those in pairs or multiple systems consisted of only RQGs (SFGs), and "mixed mergers" the rest of galaxies in the pair sample. In Figure 9, we compare the volume merger rates of dry mergers, and those of wet and mixed mergers combined, to the formation of ellipticals. Our results show that wet/mixed mergers alone 
Table 6

Mass-dependent Volume Merger Rate

\begin{tabular}{|c|c|c|c|c|c|}
\hline \multirow[t]{2}{*}{ Mass Bin } & \multicolumn{5}{|c|}{$\log \left(R_{V}\right)\left(\mathrm{Mpc}^{-3} \mathrm{dex}^{-1} \mathrm{Gyr}^{-1}\right)$} \\
\hline & $z=0$ & $0.2 \leqslant z \leqslant 0.4$ & $0.4<z \leqslant 0.6$ & $0.6<z \leqslant 0.8$ & $0.8<z \leqslant 1$ \\
\hline $9.4 \leqslant \log (M) \leqslant 9.8$ & $-3.81 \pm 0.28$ & $-3.51 \pm 0.09$ & $\cdots$ & $\cdots$ & $\cdots$ \\
\hline $9.8<\log (M) \leqslant 10.2$ & $-3.86 \pm 0.18$ & $-3.57 \pm 0.11$ & $-3.61 \pm 0.08$ & $\cdots$ & $\cdots$ \\
\hline $10.2<\log (M) \leqslant 10.6$ & $-3.81 \pm 0.10$ & $-3.25 \pm 0.10$ & $-3.59 \pm 0.09$ & $-3.34 \pm 0.08$ & $\ldots$ \\
\hline $10.6<\log (M) \leqslant 11.0$ & $-3.83 \pm 0.09$ & $-3.45 \pm 0.12$ & $-3.77 \pm 0.11$ & $-3.31 \pm 0.08$ & $-3.24 \pm 0.07$ \\
\hline $11.0<\log (M) \leqslant 11.4$ & $-4.13 \pm 0.09$ & $-3.79 \pm 0.17$ & $\cdots$ & $-3.95 \pm 0.14$ & $-3.55 \pm 0.10$ \\
\hline
\end{tabular}

can account for the formation rate of massive ellipticals and RQGs, even for the most massive ones of $M_{\text {star }} \geqslant 10^{11.3} M_{\odot}$. Our results are consistent with Lin et al. (2008), who also found that the wet and mixed mergers dominated over the dry mergers since $z \sim 1$.

It should be pointed out that ellipticals (Es) and RQGs are not identical (albeit with large overlap) since there are both red disk galaxies (Bamford et al. 2009; Bundy et al. 2010) and blue ellipticals (Kannappan et al. 2009; Huertas-Company et al. 2010). Both Es and RQGs likely originated from starforming disk galaxies: RQGs formed through SFR quenching (Bell et al. 2007; Faber et al. 2007), and ellipticals through mergers (Toomre 1978; Barnes 1988) or secular evolution (Kormendy \& Kennicutt 2004). Hopkins et al. (2008) argued that only major mergers can reproduce the kinematic properties of massive ellipticals, whereas some low mass ellipticals are "pseudo-bulges" formed through disk instabilities and secular evolution in late-type galaxies.

Many quenching mechanisms in the literature are related either directly to merger-induced feedbacks (e.g., gas consumption by extreme starbursts and gas loss due to superwinds) or to massive bulges (such as the AGN quenching, Bensen et al. 2003; Somerville et al. 2008, and morphological quenching, Martig et al. 2009). Hence, a close relation between major mergers and RQGs formation is expected. Indeed, Hopkins et al. (2008) argued that a wide range of observations favor a major-merger related quenching model to other quenching models.

Our results (Figures 8 and 9) support the model of Hopkins et al. (2008). Major mergers, dominantly wet or mixed, can fully account for the formation rates of both ellipticals and RQGs with $M_{\text {star }} \geqslant 10^{10.5} M_{\odot}$. For most massive galaxies with $M_{\text {star }} \geqslant 10^{11.3} M_{\odot}$, the major-merger rate agrees very well with the two formation rates (in this mass range most ellipticals and RQGs belong to the same population of red elliptical galaxies). In the mass range of $10^{10.5} \leqslant M_{\mathrm{star}} \leqslant 10^{11.3} M_{\odot}$, the majormerger rate is slightly higher than both formation rates. Two factors may be responsible for this. (1) Remnants of some gasrich wet mergers may remain to be blue disk galaxies (Hopkins et al. 2009), and (2) dry mergers, contributing most in this mass range, may move some red ellipticals to higher mass.

Figures 8 and 9 also show that most (i.e., $\sim 2 / 3$ ) of low mass ellipticals and RQGs $\left(M_{\text {star }} \lesssim 10^{10.3} M_{\odot}\right)$ are not produced by mergers. Many authors have argued that these galaxies are mostly quenched satellite galaxies whose gas halos are stripped by much more massive central galaxies (van den Bosch et al. 2008; Peng et al. 2010).

\subsection{Impacts of Major Mergers on Galaxy Assembly}

Mergers shift galaxies from lower mass bins to higher mass bins in the GSMF. The efficiency of this process is clearly critical for the hierarchical structure formation paradigm. Drory \&
Alvares (2008) tried to address this issue via comparisons between observed GSMF variation against redshift and that predicted by the SFR versus $z$ relation which was well established (in particular for $z \leqslant 1$ ), attributing the difference to the merger effects (including both major and minor mergers). Our results on major-merger rates provide a new and more direct approach.

We define the following parameter $\hat{\phi}_{\text {merger }}$ to evaluate the impact of major mergers on the GSMF $\phi$ :

$$
\hat{\phi}_{\text {merger }}\left(M_{\text {star }}\right)=R_{V}\left(M_{\text {star }}\right) / \phi\left(M_{\text {star }}\right)-R_{\text {mg }}\left(M_{\text {star }}\right),
$$

where $\phi\left(M_{\text {star }}\right)$ is the GSMF (D09), $R_{V}\left(M_{\text {star }}\right)$ is the volume merger rate defined in Equation (12), and $R_{\mathrm{mg}}$ is the differential merger rate estimated using Equation (11).

In Figure 10, we compare our results with those of Drory \& Alvares (2008). The solid line is our result on the mean $\hat{\phi}_{\text {merger }}\left(M_{\text {star }}\right)$ over $0.3<z<0.9$. In this redshift range, major mergers (as opposed to star formation) have significant impact on the galaxy mass assembly only for the most massive galaxies with $M_{\text {star }} \gtrsim 10^{11.3}$. The GSMF change due to major mergers dominates that due to star formation at $M_{\text {star }} \gtrsim 10^{11.3}$. For less massive galaxies with $M_{\text {star }}<10^{11.2}$ the GSMF change due to major mergers is negligible (amplitude $\leqslant 10 \% \mathrm{Gyr}^{-1}$ ) in comparison to that due to star formation. For these galaxies, our result is much flatter than that of Drory \& Alvares (2008) for the GSMF change rate due to mergers at both $z=0.5$ and $z=1$. For massive galaxies $\left(M_{\text {star }} \gtrsim 10^{11.3}\right)$, we find much steeper mass dependence than Drory \& Alvares (2008). The major reason for the discrepancy is due to the difference between the redshiftdependent GSMFs used in this work (Drory et al. 2009) and those in Drory \& Alvares (2008); the latter were derived using data from earlier FDF/GOODS surveys.

\section{MAJOR MERGERS AND (U)LIRGs}

Kartaltepe et al. (2010) carried out a study of LIRGs $\left(10^{11}<\right.$ $\left.L_{\mathrm{IR}} / L_{\odot}<10^{12}\right)$ and $(\mathrm{U})$ LIRGs $\left(L_{\mathrm{IR}} / L_{\odot}>10^{12}\right)$ in the S-COSMOS survey (Sanders et al. 2007). We neglect (U)LIRGs of $L_{\mathrm{IR}} / L_{\odot}>10^{12.5}$ since very few galaxies have such high IR luminosities, and the AGN fraction increases rapidly with the $L_{\mathrm{IR}}$ among these galaxies (Kartaltepe et al. 2010). Taking galaxies with $10^{11.5}<L_{\mathrm{IR}} / L_{\odot}<10^{12.5}$ from their sample and adopting a (U)LIRG timescale of $140 \mathrm{Myr}$ (Kartaltepe et al. 2010), we estimate the (U)LIRG rates in different stellar mass bins and in the redshift range of $0.2<z<1.0$. The results are presented in Figure 11, compared with the average mass-dependent volume merger rate.

The mass dependence of (U)LIRG rates in all photo- $z$ bins has the shape of the log-normal function, peaking at a rather constant mass of $\sim \log \left(M_{\text {star }}^{*} / M_{\odot}\right)=10.8$. In the low photo- $z$ bin $(0.2 \leqslant z \leqslant 0.4)$ (U)LIRGs are less frequent, consistent with the fact that (U)LIRGs are very rare in the local universe 

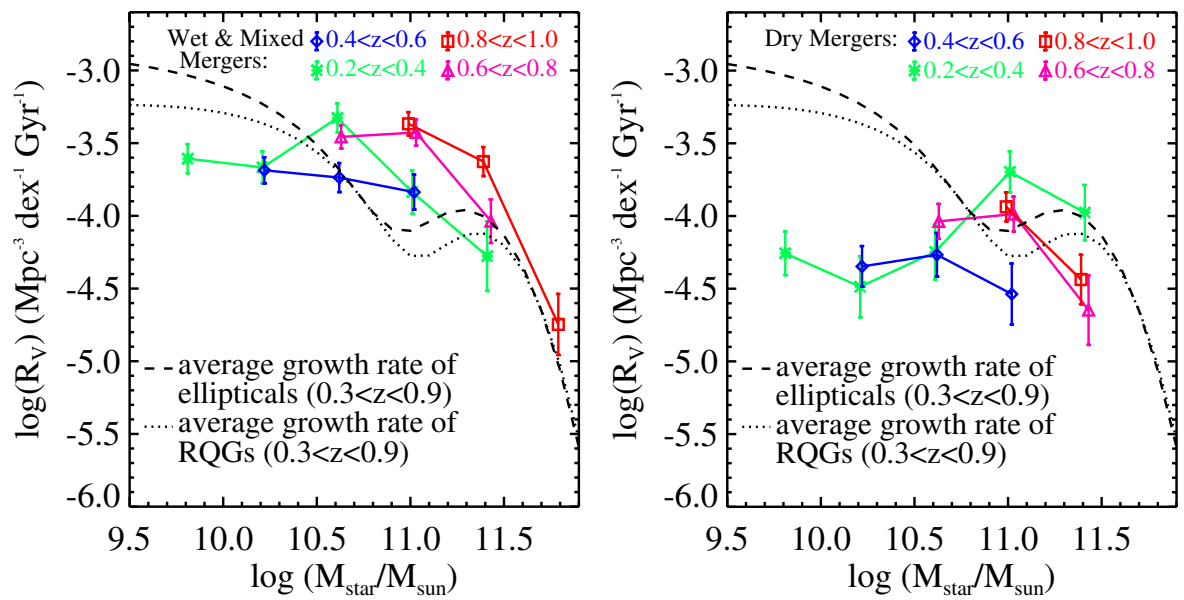

Figure 9. Left: mass-dependent volume merger rates of wet $(\mathrm{S}+\mathrm{S})$ and mixed $(\mathrm{S}+\mathrm{E})$ mergers, in different photo- $z$ bins. The dashed line is the average growth rate of ellipticals between $z=0.3-0.9$ and dotted line that of RQGs, both being estimated using results of Ilbert et al. (2010). Right: mass-dependent volume merger rates of dry (E+E) mergers.

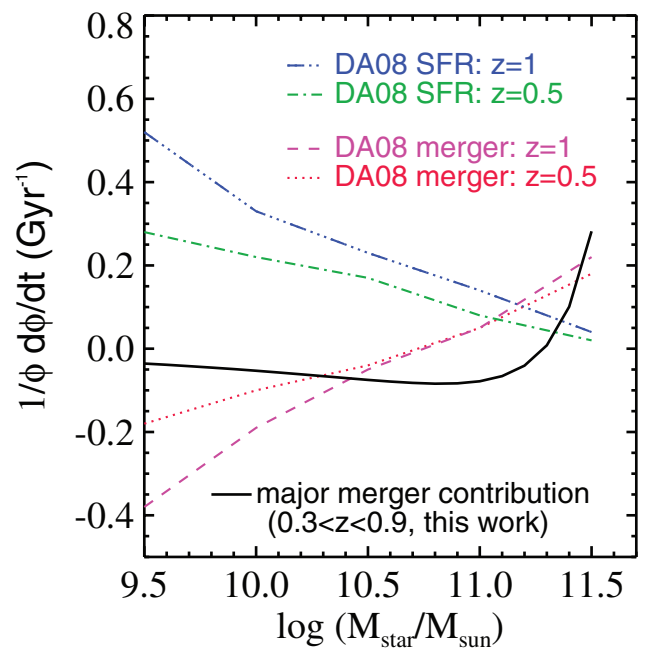

Figure 10. Fractional change rate of the GSMF. The solid line is our result on the mean $\hat{\phi}_{\text {merger }}$ (contribution of major mergers, see Equation (13)) over $0.3<z<0.9$. The dotted and dashed lines are the results of Drory \& Alvares (2008) on the contribution of mergers (including both major and minor mergers) at $z=0.5$ and $z=1$, respectively. The dot-dashed and triple-dot-dashed lines are the results of Drory \& Alvares (2008) on the contribution of star formation at $z=0.5$ and $z=1$, respectively.

(Sanders \& Mirabel 1996). The average mass-dependent volume merger rate is above or comparable to the (U)LIRG rates in all redshift and mass bins, and therefore it is possible that most of the (U)LIRGs in this redshift range are major mergers, just like their local counterparts (Sanders \& Mirabel 1996). Using morphological classifications, Kartaltepe et al. (2010) found that $\gtrsim 50 \%$ of their (U)LIRGs are major mergers. This means that the merger-induced (U)LIRG rates are even more below the average merger rate, in particular for $M_{\text {star }} \lesssim 10^{10.3}$ and $M_{\text {star }} \gtrsim 10^{11.3}$, than are depicted in Figure 11. Hence, it is likely that a large fraction of major mergers, in particular those with $M_{\text {star }} \lesssim 10^{10.3}$ or $M_{\text {star }} \gtrsim 10^{11.3}$, may not become (U)LIRGs. It is interesting to note that the most massive mergers of $M_{\text {star }} \gtrsim 10^{11.3} M_{\odot}$ have rather low (U)LIRG rates. Most of them are wet or mixed mergers, but many probably have relatively low gas content. Galaxies of lower mass $\left(M_{\text {star }} \sim 10^{10} M_{\odot}\right)$ also have low (U)LIRG rates because their gas mass is not

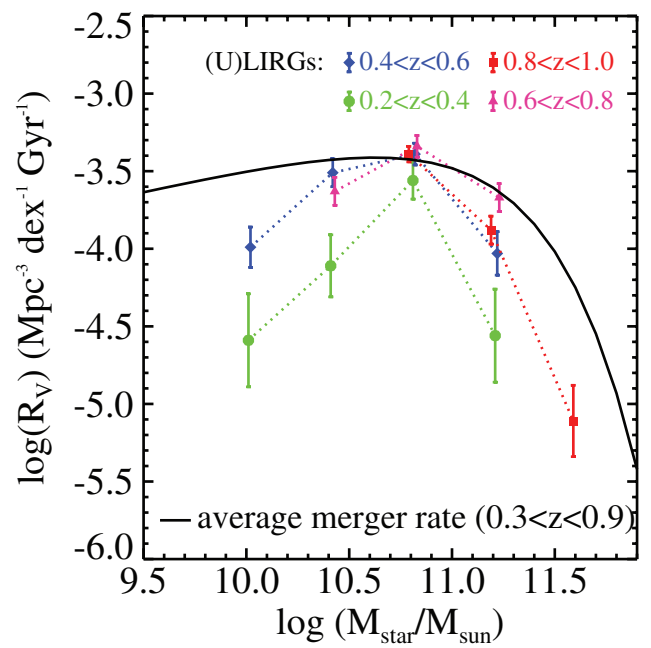

Figure 11. Volume rates of (U)LIRGs $\left(10^{11.5}<L_{\mathrm{IR}} / L_{\odot}<10^{12.5}\right)$, in different redshift and stellar mass bins. Estimated using data taken from Kartaltepe et al. (2010) and assuming a (U)LIRG timescale of $140 \mathrm{Myr}$. The solid line is the average mass-dependent volume merger rate (this work), identical to that in Figure 8.

adequate to sustain the very high SFR of the extreme starbursts in (U)LIRGs.

\section{SUMMARY}

We have presented results of a statistical study on the cosmic evolution of the mass-dependent major-merger rate since $z=$ 1. A stellar mass limited sample of major-merger pairs (the CPAIR sample) was selected from the archive of the COSMOS survey. It includes 617 galaxies in pairs/multiple systems with stellar mass ratios $\mu \leqslant 2.5$, projected separations in the range of $5 h^{-1} \mathrm{kpc} \leqslant r_{\text {proj }} \leqslant 20 h^{-1} \mathrm{kpc}$, and in the photo- $z$ range of $0.2 \leqslant z_{\text {phot }} \leqslant 1.0$. The pair selection was based on photo- $z$, with the criterion of $\Delta z_{\text {phot }} /\left(1+z_{\text {phot }}\right) \leqslant 0.03$, and on visual inspections of the $H S T$-ACS images. The CPAIR sample is divided into four photo- $z$ bins of $[0.2 \leqslant z \leqslant 0.4$, $0.4<z \leqslant 0.6,0.6<z \leqslant 0.8,0.8<z \leqslant 1$ ]. Various biases in the sample selection that caused incompleteness and spurious pair contaminations have been studied. This resulted in a completeness correction factor of $[0.78 \pm 0.05$, $0.74 \pm 0.05,0.71 \pm 0.05,0.60 \pm 0.05]$ and a reliability correction 
factor of $[0.80 \pm 0.06,0.79 \pm 0.06,0.77 \pm 0.06,0.78 \pm 0.06]$, respectively, for pairs in the four photo- $z$ bins. The CPAIR sample is complemented by a local $(z=0)$ major-merger pair sample, selected in the $K$ band from cross matches between 2MASS and SDSS-DR5 (an updated version of the KPAIR sample in Domingue et al. 2009).

Mass-dependent pair fractions at different redshifts derived using CPAIR and KPAIR samples show no significant variations with stellar mass. The integral pair fraction (i.e., pair fraction of all galaxies regardless of stellar mass) demonstrates a moderately strong cosmic evolution, with the best-fitting function of $f_{\text {pair }}=10^{-1.88( \pm 0.03)}(1+z)^{2.2( \pm 0.2)}$.

The merger timescale was taken from the simulation results of Lotz et al. (2010): $T_{\mathrm{mg}} / \mathrm{Gyr}=0.3 \times\left(M_{\mathrm{star}} / 10^{10.7} M_{\odot}\right)^{-0.3}(1+$ $z / 8)$. The best-fitting function for the differential merger rate (for $\mu \leqslant 3$ mergers) is $R_{\mathrm{mg}} / \mathrm{Gyr}^{-1}=0.053 \times$ $\left(M_{\text {star }} / 10^{10.7} M_{\odot}\right)^{0.3}(1+z)^{2.2} /(1+z / 8)$. Accordingly, on average, galaxies of $M_{\text {star }} \sim 10^{10}-10^{11.5} M_{\odot}$ have undergone $\sim 0.5-1.5$ times major mergers since $z=1$. Our result on the differential merger rate is in very good agreement with that estimated using morphologically selected major mergers (Conselice et al. 2009) and with the prediction of the "semi-empirical" model of Hopkins et al. (2010a) and that of the high-resolution $\Lambda$ CDM $N$-body simulation of Stewart et al. (2009).

The mass-dependent major-merger rates derived in this work indicate that, for massive galaxies $\left(M_{\text {star }} \geqslant 10^{10.5} M_{\odot}\right)$ at $z \leqslant 1$, major mergers involving SFGs (i.e., wet and mixed mergers) can fully account for the formation rates of both ellipticals and RQGs, lending support to models that link both bulge formation and SFR quenching to major mergers (e.g., Hopkins et al. 2008). On the other hand, major mergers cannot be responsible for the formation of most low mass ellipticals and RQGs of $M_{\text {star }} \lesssim 10^{10.3} M_{\odot}$. Dry mergers contribute negligibly to the major-merger rate in all mass and photo- $z$ bins. Major mergers have significant impact on the stellar mass assembly of the most massive galaxies $\left(M_{\text {star }} \geqslant 10^{11.3} M_{\odot}\right)$. For less massive galaxies the stellar mass assembly is dominated by the star formation.

Comparisons with mass-dependent (U)LIRG rates in different redshift bins suggest that the frequency of major-merger events is comparable to or higher than that of (U)LIRGs. Most low mass mergers $\left(M_{\text {star }} \lesssim 10^{10.3} M_{\odot}\right)$ and most very massive mergers $\left(M_{\text {star }} \gtrsim 10^{11.3} M_{\odot}\right)$ may not become (U)LIRGs.

This work is based on observations with the NASA/ESA Hubble Space Telescope, obtained at the Space Telescope Science Institute, which is operated by AURA, Inc., under NASA contract NAS 5-26555; and the Spitzer Space Telescope, which is operated by the Jet Propulsion Laboratory, California Institute of Technology under NASA contract 1407; also based on data collected at the Subaru Telescope, which is operated by the National Astronomical Observatory of Japan; the XMM-Newton, an ESA science mission with instruments and contributions directly funded by ESA Member States and NASA; the European Southern Observatory under Large Program 175.A-0839, Chile; Kitt Peak National Observatory, Cerro Tololo Inter-American Observatory, and the National Optical Astronomy Observatory, which are operated by the Association of Universities for Research in Astronomy (AURA), Inc., under cooperative agreement with the National Science Foundation; the National Radio Astronomy Observatory which is a facility of the National Science Foundation operated under cooperative agreement by Associated Universities, Inc.; and the Canada-France-Hawaii Telescope with MegaPrime/MegaCam operated as a joint project by the CFHT

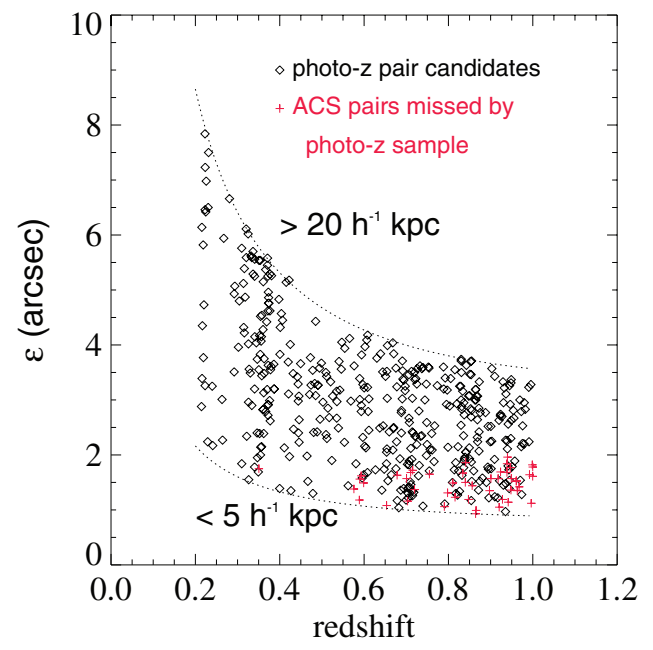

Figure 12. Plot of the angular separation $\epsilon$ vs. redshift relation for pair candidates (open diamonds), and for very close ACS pairs that are missed by the photo- $z$ pair candidate sample (plus symbols).

(A color version of this figure is available in the online journal.)

Corporation, CEA/DAPNIA, the NRC and CADC of Canada, the CNRS of France, TERAPIX and the University of Hawaii. C.K.X. acknowledges Kevin Bundy for constructive discussions and Alexie Leauthaud for help in analyzing the COSMOS HST-ACS lensing catalog. Zara Scoville is thanked for proofing the English of the manuscript. Y.Z. and Y.G. are grateful for the financial support from the NSF of China (grant Nos. 10833006 and 10903029). Y.Z. thanks IPAC for the hospitality and the financial support during his visit.

\section{APPENDIX A}

\section{INCOMPLETENESS DUE TO MISSING VERY CLOSE PAIRS-ANALYSIS}

In order to estimate how many pairs with $\epsilon \lesssim 2^{\prime \prime}$ are missing in our sample, we carried out an analysis exploiting the COSMOS HST-ACS lensing catalog (Leauthaud et al. 2007, 2010). It includes $1.2 \times 10^{6}$ galaxies detected by HST-ACS in the F814 band (hereafter $i_{\mathrm{ACS}}$ band), with an angular resolution of FWHM =0 0.'12 (Leauthaud et al. 2007). From this catalog, we selected a sample of "very close ACS pairs" through the following procedure.

1. Find the match in the $H S T$-ACS lensing catalog for every D09 galaxy of $\log \left(M_{\text {star }}\right) \geqslant \log \left(M_{\text {lim }}\right)$ (see Section 2 for the definition of $\left.M_{\mathrm{lim}}\right)$ with a searching radius of 0.8 and the criterion of $\left|i_{\mathrm{ACS}}-i_{\mathrm{AB}, \mathrm{D} 09}\right| \leqslant 1$ mag.

2. Around the ACS matches of D09 galaxies, we search for ACS pairs with three criteria: (1) $\left|\Delta i_{\mathrm{ACS}}\right| \leqslant 1 \mathrm{mag}$, (2) $\epsilon<2^{\prime \prime}$. 0 , and (3) $r_{\text {proj }} \geqslant 5 h^{-1} \mathrm{kpc}$.

The procedure selected 222 very close ACS pairs. Among them, 171 pairs have both component galaxies with photo- $z$ matches, including 53 pairs found in the photo- $z$ pair sample and the remaining 118 pairs consisting of galaxies of discordant photo- $z$ or with mass ratios larger than 2.5. In the remaining 51 pairs, 4 are multi-peak single galaxies in the ACS images. The final sample has 47 pairs in which only one of two galaxies was detected in the photo- $z$ catalog. These pairs, shown in Figure 12 by red crosses, have the average angular separation $\langle\epsilon\rangle=1$ ". 45 with the standard deviation of $\sigma=0$ '.27. Figure 12 also shows that, for pairs of $z>0.5$, the lower boundary for the 


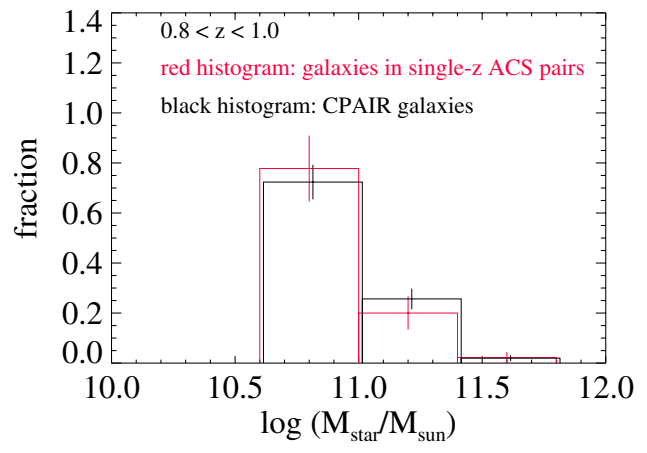

Figure 13. Comparison of mass distributions of CPAIR galaxies and of galaxies in the single photo- $z$ ACS pairs $\left(\epsilon<2^{\prime \prime}\right)$ with $0.8<z_{\text {phot }}<1.0$, including 31 of the 47 "very close" ACS pairs.

(A color version of this figure is available in the online journal.)

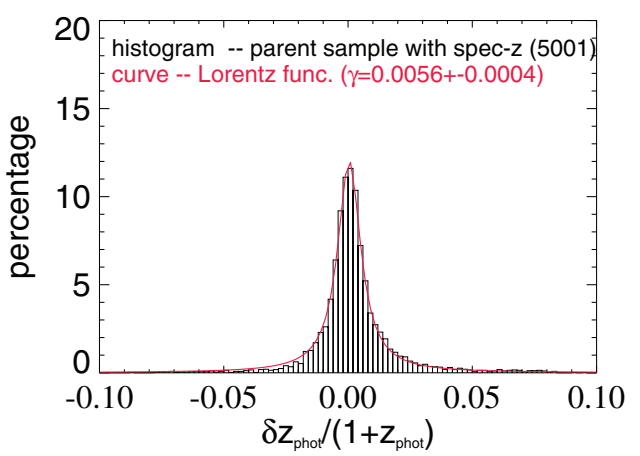

Figure 14. Plot of $\left(z_{\text {phot }}-z_{\text {spec }}\right) /\left(1+z_{\text {spec }}\right)$ distribution of 5001 galaxies in the parent sample that have spec- $z$. The red solid curve is the best-fit Lorentzian function.

(A color version of this figure is available in the online journal.)

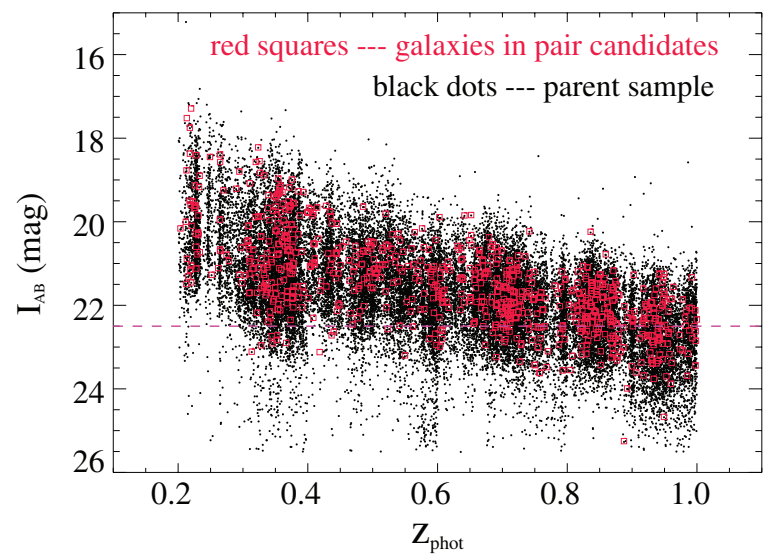

Figure 15. $i$-band magnitude $\left(i_{\mathrm{AB}}\right)$ vs. photo- $z$ plot. Galaxies in the pair candidates are shown by red squares, and galaxies in the parent sample (Table 1) by black dots. The dashed line marks the boundary of $i_{\mathrm{AB}}=22.5$.

(A color version of this figure is available in the online journal.)

pair separations, $r_{\text {proj }} \geqslant 5 h^{-1} \mathrm{kpc}$, corresponds to an angular separation of $\epsilon \sim 1^{\prime \prime}$.

We then used Monte Carlo simulations to estimate the expected number of spurious pairs in the sample of very close ACS pairs, utilizing the 138,001 galaxies in D09 sample. In each of the 100 simulations, we randomly put these galaxies in a $1.7 \mathrm{deg}^{2}$ region, with all other properties of the galaxies, including the photo- $z$ and stellar mass, intact. We then search companions around each of the galaxies of $\log \left(M_{\text {star }}\right) \geqslant \log \left(M_{\text {lim }}\right)$ in the simulated sample according to the following criteria: $\left|\Delta i_{\mathrm{AB}}\right| \leqslant 1$

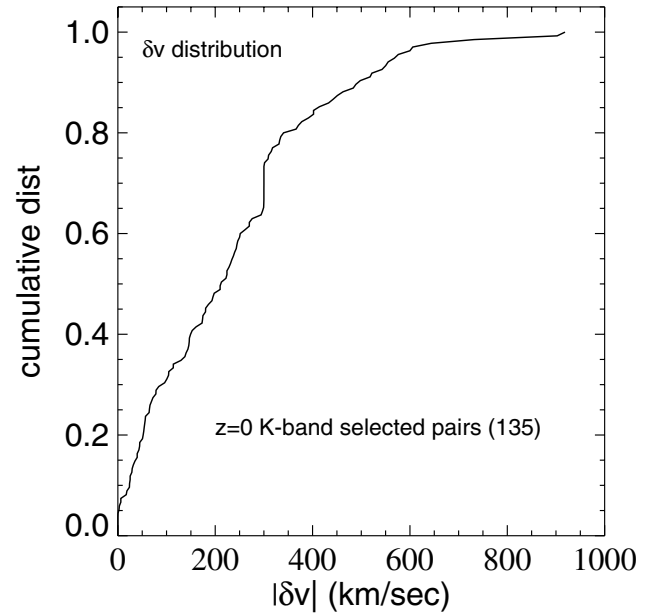

Figure 16. Cumulative distribution of $\Delta v$ of major-merger pairs, derived using a sample of $z=0 K$-band-selected pairs (Domingue et al. 2009).

mag, (2) $\epsilon<2$ '. 0 , and (3) $r_{\text {proj }} \geqslant 5 h^{-1} \mathrm{kpc}$. Spurious pairs that do not pass the four pair selection criteria in Section 2 were then counted. These simulations found a mean spurious pair number of 103.3 with a $1 \sigma$ dispersion of 7.0. As described above, the number of confirmed spurious pairs of photo- $z$ galaxies in the sample of very close ACS pairs is 118 . This number is slightly higher than the mean total number of spurious pairs $(103.3 \pm 7.0)$ predicted by the Monte Carlo simulations, perhaps due to galaxy clustering. Thus, the majority of 47 single photo- $z$ ACS pairs are in fact real and not chance superpositions. Indeed their ACS images very often show signs of interaction. In what follows we shall make the conservative assumption that all 47 ACS pairs are real major-merger pairs.

There are $N_{\mathrm{ACS}}=[1,5,10,31]$ single photo- $z$ ACS pairs in the four photo- $z$ bins. The corresponding incompleteness due to missing of very close pairs, estimated according to the ratio $N_{\text {ACS }} /\left(N_{\text {photo }-} z+N_{\text {ACS }}\right)\left(N_{\text {photo }-} z\right.$ being the number of photo$z$ pair candidates), is $0.01 \pm 0.01,0.06 \pm 0.03,0.08 \pm 0.03$, and $0.20 \pm 0.04$ for the four photo- $z$ bins, respectively. As a check, we found no significant difference between the stellar mass distributions of galaxies in the ACS single photo- $z$ pairs and of those in the CPAIR sample in the redshift bin of $0.8<z_{\text {phot }}<1.0$ (Figure 13).

\section{APPENDIX B}

\section{INCOMPLETENESS DUE TO PHOTO- $z$ ERRORS AND SPURIOUS PAIRS DUE TO PROJECTION-MONTE CARLO SIMULATIONS}

We examine the accuracy of the photo-z's by comparing them with the spectroscopically measured redshift (hereafter spec$z$ 's) found in the archive of the zCOSMOS-bright survey (Lilly et al. 2007), which is a magnitude-limited survey $\left(i_{\mathrm{AB}}<22.5\right)$ of intermediate depth $(0.1<z<1.2)$. The archive includes space-z's of 10,643 galaxies in the $1.7 \mathrm{deg}^{2}$ COSMOS field. There are 5001 matches (matching radius $=2^{\prime \prime}$ ) between galaxies in the parent sample (Table 1) and zCOSMOS sources with reliable spec- $z$ measurements (z-class indices being 4's, 3 's, $9.5,9.4,9.3,2.5,2.4$, or 1.5 ). This is $19.5 \%$ of 25,711 galaxies in the parent sample that have $i_{\mathrm{AB}}<22.5$. Figure 14 shows $\left(z_{\text {phot }}-z_{\text {spec }}\right) /\left(1+z_{\text {spec }}\right)$ of the 5001 galaxies. The distribution, an estimate of the photo- $z$ error distribution (the spec- $z$ error $\lesssim 100 \mathrm{~km} \mathrm{~s}^{-1}$; Lilly et al. 2007), has a $1.48 \times$ 
Table 7

Pairs in zCOSMOS Survey (ZPAIR)

\begin{tabular}{|c|c|c|c|c|c|c|c|}
\hline $\begin{array}{l}\text { ZPAIR } \\
\text { ID } \\
\end{array}$ & $\begin{array}{c}\Delta v_{\text {spec }} \\
\left(\mathrm{km} \mathrm{s}^{-1}\right)\end{array}$ & $\begin{array}{l}\text { R.A.1 } \\
\text { (deg) }\end{array}$ & $\begin{array}{c}\text { Decl.1 } \\
(\mathrm{deg})\end{array}$ & $z_{\text {spec }, 1}$ & Z-class 1 & $z_{\text {phot, } 1}$ & $\begin{array}{c}\log \left(M_{\mathrm{star}, 1}\right) \\
\left(M_{\odot}\right) \\
\end{array}$ \\
\hline & $\Delta z_{\text {phot }} /\left(1+z_{\text {phot }}\right)$ & $\begin{array}{l}\text { R.A.2 } \\
\text { (deg) }\end{array}$ & $\begin{array}{c}\text { Decl.2 }_{.2} \\
(\mathrm{deg})\end{array}$ & $z_{\text {spec }, 2}$ & z-class 2 & $z_{\text {phot }, 2}$ & $\begin{array}{c}\log \left(M_{\text {star }, 2}\right) \\
\left(M_{\odot}\right) \\
\end{array}$ \\
\hline \multirow[t]{2}{*}{01} & 43 & 149.713610 & 2.019610 & 0.6444 & 4.5 & 0.6518 & 10.92 \\
\hline & 0.012 & 149.713950 & 2.019773 & 0.6441 & 4.5 & 0.6324 & 10.83 \\
\hline \multirow[t]{2}{*}{02} & 218 & 149.839700 & 1.929228 & 0.3722 & 3.5 & 0.3856 & 10.80 \\
\hline & 0.020 & 149.838780 & 1.930125 & 0.3711 & 4.5 & 0.3585 & 10.76 \\
\hline \multirow[t]{2}{*}{$03^{\mathrm{a}}$} & 70 & 150.009050 & 2.274964 & 0.4726 & 2.5 & 0.5769 & 11.05 \\
\hline & 0.071 & 150.008210 & 2.275954 & 0.4730 & 2.5 & 0.4645 & 10.78 \\
\hline \multirow[t]{2}{*}{$04^{\mathrm{b}}$} & 817 & 150.107580 & 2.556516 & 0.5038 & 3.5 & 0.4915 & 10.33 \\
\hline & 0.004 & 150.107510 & 2.557509 & 0.4990 & 3.5 & 0.4969 & 10.21 \\
\hline \multirow[t]{2}{*}{05} & 73 & 150.115480 & 1.975120 & 0.4385 & 4.5 & 0.4431 & 10.45 \\
\hline & 0.025 & 150.115680 & 1.976216 & 0.4381 & 3.5 & 0.4069 & 10.05 \\
\hline \multirow[t]{2}{*}{06} & 65 & 150.126060 & 1.913758 & 0.7360 & 2.5 & 0.7168 & 11.17 \\
\hline & 0.003 & 150.125690 & 1.913726 & 0.7365 & 3.5 & 0.7108 & 11.15 \\
\hline \multirow[t]{2}{*}{$07^{\mathrm{a}}$} & 11 & 150.168760 & 2.315481 & 0.8524 & 2.5 & 0.7921 & 10.97 \\
\hline & 0.031 & 150.168890 & 2.316234 & 0.8523 & 1.5 & 0.8473 & 10.94 \\
\hline \multirow[t]{2}{*}{08} & 220 & 150.196380 & 2.371582 & 0.6834 & 4.5 & 0.6783 & 10.86 \\
\hline & 0.003 & 150.196460 & 2.370591 & 0.6850 & 22.5 & 0.6726 & 10.64 \\
\hline \multirow[t]{2}{*}{09} & 103 & 150.230880 & 1.845002 & 0.6226 & 2.5 & 0.6072 & 10.67 \\
\hline & 0.014 & 150.230550 & 1.844713 & 0.6233 & 3.5 & 0.5840 & 10.60 \\
\hline \multirow[t]{2}{*}{10} & 118 & 150.258800 & 1.988773 & 0.7258 & 2.5 & 0.7168 & 10.70 \\
\hline & 0.001 & 150.258510 & 1.988547 & 0.7267 & 2.5 & 0.7191 & 10.32 \\
\hline \multirow[t]{2}{*}{$11^{\mathrm{b}}$} & 6318 & 150.359560 & 2.659517 & 0.4309 & 22.5 & 0.3991 & 10.94 \\
\hline & 0.002 & 150.359280 & 2.660543 & 0.3974 & 3.5 & 0.4018 & 10.81 \\
\hline \multirow[t]{2}{*}{12} & 142 & 150.396810 & 2.519130 & 0.2189 & 24.5 & 0.2283 & 9.97 \\
\hline & 0.003 & 150.396980 & 2.517332 & 0.2195 & 4.5 & 0.2323 & 9.93 \\
\hline \multirow[t]{2}{*}{13} & 333 & 150.421160 & 2.654335 & 0.2144 & 4.5 & 0.2312 & 9.57 \\
\hline & 0.003 & 150.421480 & 2.654870 & 0.2158 & 4.5 & 0.2271 & 9.21 \\
\hline \multirow[t]{2}{*}{14} & 118 & 150.457210 & 2.695287 & 0.2189 & 4.5 & 0.2277 & 9.45 \\
\hline & 0.008 & 150.456970 & 2.696160 & 0.2194 & 4.5 & 0.2184 & 9.07 \\
\hline \multirow[t]{2}{*}{15} & 219 & 150.494640 & 2.187936 & 0.3695 & 4.5 & 0.3674 & 9.97 \\
\hline & 0.007 & 150.494120 & 2.187388 & 0.3684 & 3.5 & 0.3772 & 9.58 \\
\hline \multirow[t]{2}{*}{16} & 196 & 150.504990 & 2.225083 & 0.8374 & 22.5 & 0.8360 & 11.72 \\
\hline & 0.002 & 150.504730 & 2.224246 & 0.8357 & 2.5 & 0.8315 & 11.34 \\
\hline
\end{tabular}

Notes.

a Missing in the CPAIR sample.

b Spurious pairs (with $\Delta v_{\text {spec }}>500 \mathrm{~km} \mathrm{~s}^{-1}$ ) in the CPAIR sample.

$\operatorname{median}\left(\left|z_{\text {phot }}-z_{\text {spec }}\right| /\left(1+z_{\text {spec }}\right)\right)=0.007$, consistent with the result of Ilbert et al. (2009). It can be best fitted by a Lorentzian function of the form

$$
L(x)=\frac{1}{\gamma \pi} \frac{1}{1+\left(\left(x-x_{0}\right) / \gamma\right)^{2}},
$$

with $x=\left(z_{\text {phot }}-z_{\text {spec }}\right) /\left(1+z_{\text {spec }}\right), \gamma=0.0056 \pm 0.0004$ and $x_{0}=0.0005 \pm 0.0003$.

In Figure 15, the $i$-band magnitudes are shown for galaxies in pair candidates (red squares) and in the parent sample (black dots). Both the pair sample and the parent sample are dominated by galaxies brighter than $i_{\mathrm{AB}}=22.5$. In the four redshift bins of the sample of pair candidates, fractions of $0.032,0.061,0.168$, and 0.352 of the sample are fainter than $i_{\mathrm{AB}}=22.5$ for the four redshift bins.

The Monte Carlo simulations for the estimation of completeness correction factor due to photo- $z$ errors were based on the above results. The simulations included a total of 1000 repeats for each photo- $z$ bin, each containing 100 pairs. For pairs in a given photo- $z$ bin, each galaxy had a chance to be fainter (or brighter) than $i_{\mathrm{AB}}=22.5$, the probability (simulated by a random number generator) being equal to the observed fraction of such galaxies in the photo- $z$ bin. For galaxies brighter (fainter) than $i_{\mathrm{AB}}=22.5$, an error was assigned to the variable $z_{\text {phot }} /\left(1+z_{\text {phot }}\right)$ using a random number generator with weighted probability distribution function given by a Lorentzian with $x_{0}=0$ and $\gamma_{\text {brt }}=0.0056\left(\gamma_{\mathrm{fnt}}=0.0096\right)$. The value of $\gamma_{\text {fnt }}$ is derived from $\gamma_{\text {brt }} \times 0.012 / 0.007$, where $0.012 / 0.007$ is the ratio between the photo- $z$ accuracies for galaxies of $22.5 \leqslant i_{\mathrm{AB}}<24$ and of $i_{\mathrm{AB}}<22.5$ (Ilbert et al. 2009). We assumed that the photo- $z$ difference in a pair being purely due to photo- $z$ errors, ignoring the real recession velocity difference. The completeness factor was estimated by the fraction of simulated pairs with $\left|\Delta z_{\text {phot }} /\left(1+z_{\text {phot }}\right)\right| \leqslant 0.03$. 

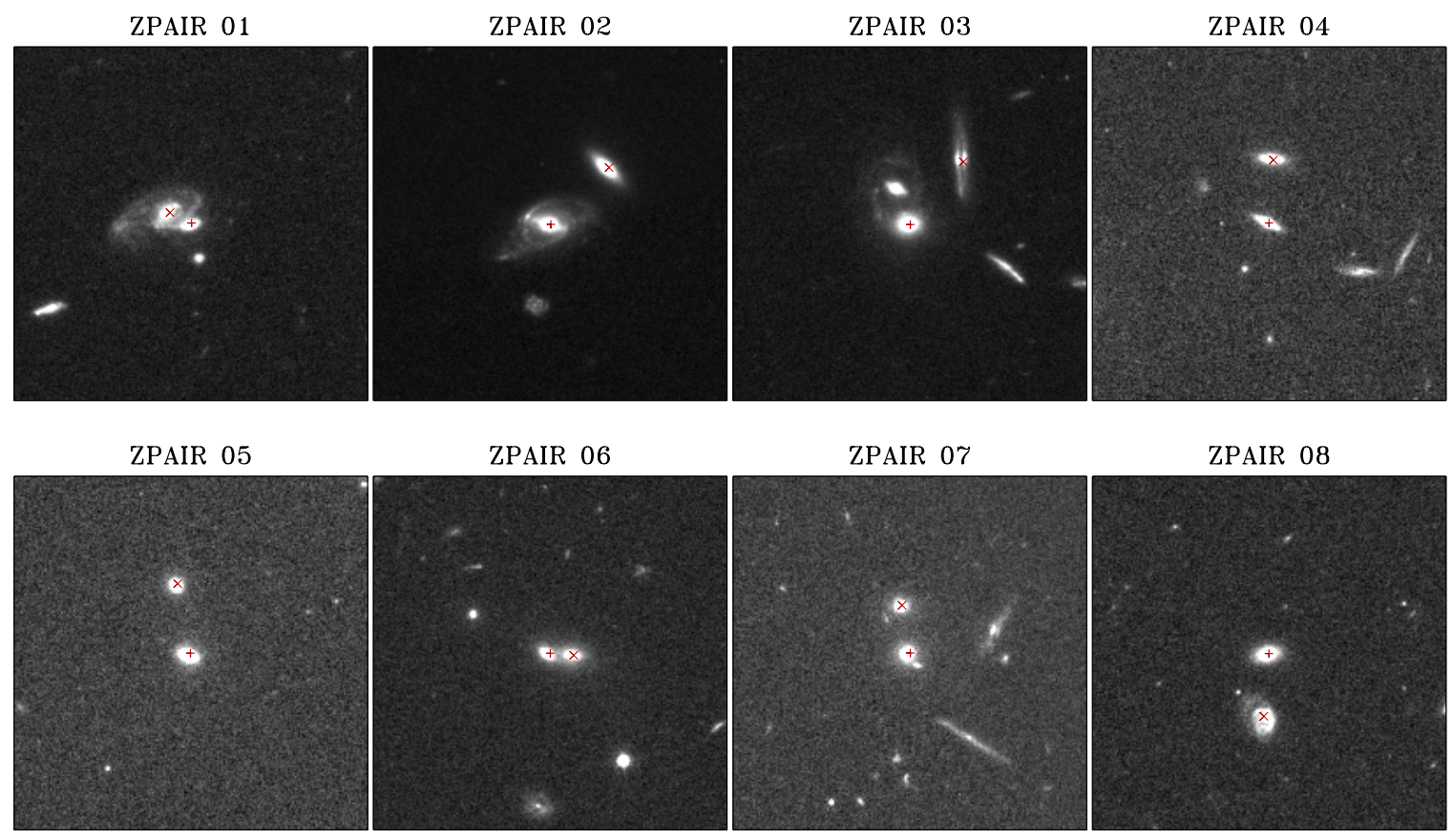

ZPAIR 08

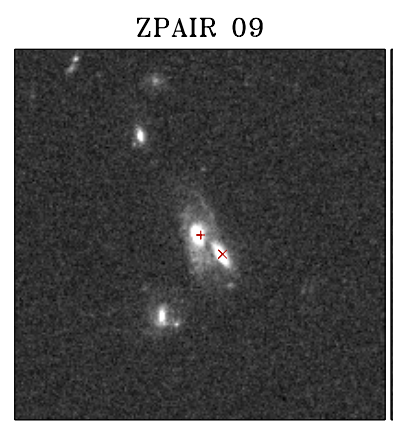

ZPAIR 10

ZPAIR 11
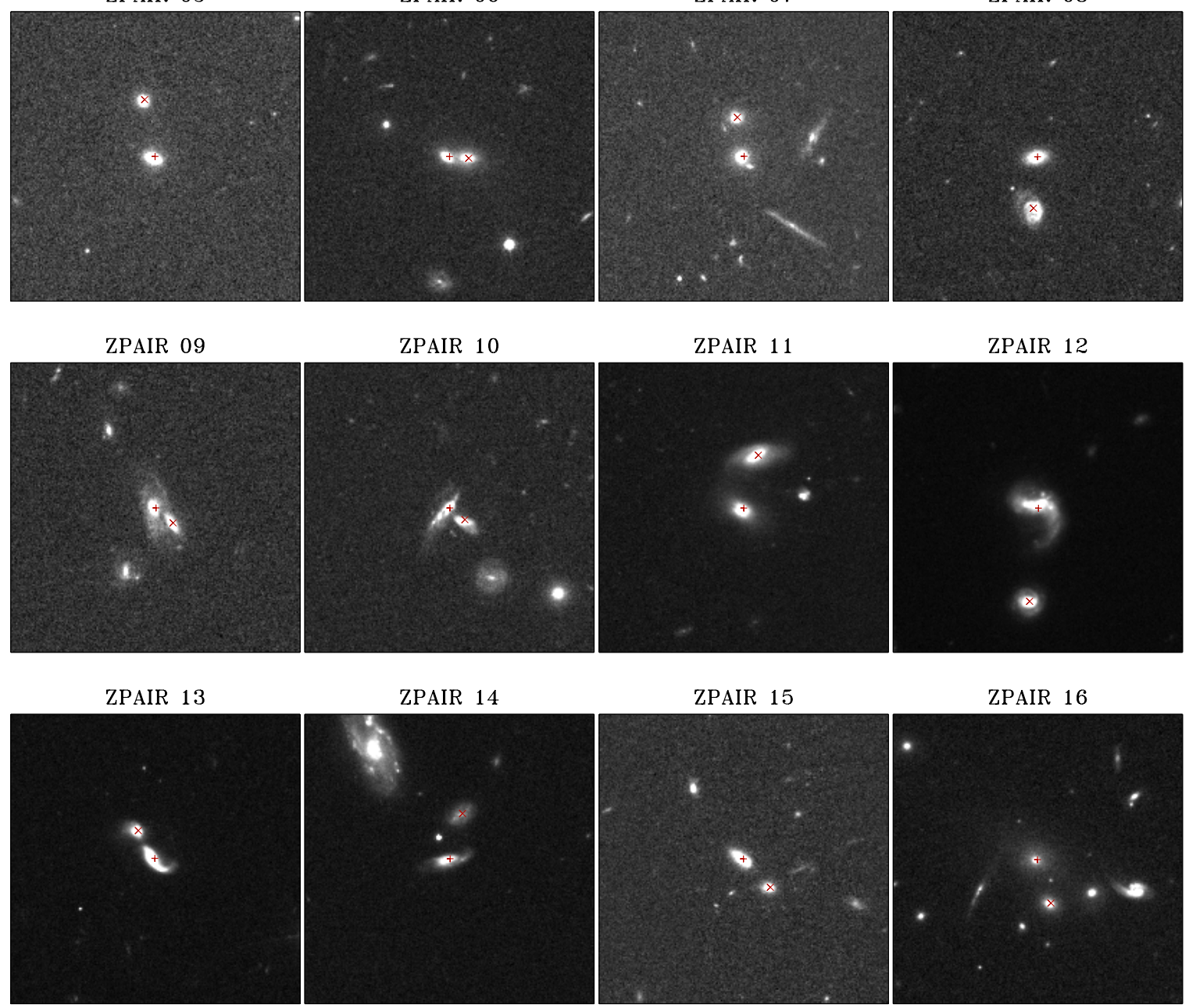

Figure 17. HST-ACS (F814) images of close major-merger pairs in the zCOSMOS survey (ZPAIRs in Table 7). The size of all images is $20^{\prime \prime} \times 20^{\prime \prime}$. The crosses mark the positions of component galaxies in the pairs. Notes: ZPAIR-03 and ZPAIR-07 are not in the CPAIR (photo- $z$ pairs) sample. The large neighbor in ZPAIR-14 is likely a foreground galaxy. ZPAIR-16 is in a compact group of galaxies (three in this group were included in the CPAIR sample).

(A color version of this figure is available in the online journal.)

According to the simulations, the completeness correction factor is $[0.78 \pm 0.05,0.77 \pm 0.06,0.76 \pm 0.05,0.73 \pm 0.05]$ in the four photo- $z$ bins.

Another set of Monte Carlo simulations (each consisting of 1000 repeats) was carried out to estimate the SPF due to projection, utilizing the 138,001 galaxies in the D09 sample. Here we pretended that the photo-z's in that sample are $100 \%$ accurate, and then added errors to them using the same algorithm as described above. The sky coordinates of the galaxies were also randomized, filling a $1.7 \mathrm{deg}^{2}$ region uniformally. The other properties of the galaxies, includ- ing the stellar mass, were left intact. We then selected pairs from this simulated parent sample by applying the four selection criteria presented in Section 2. Spurious pairs were identified when the "true velocity difference," calculated using the "true redshifts" (i.e., the photo- $z$ 's without added error), is $>500 \mathrm{~km} \mathrm{~s}^{-1}$. In the four photo- $z$ bins, the simulations found $[7.5 \pm 2.8,6.2 \pm 2.5,11.4 \pm 3.3,11.1 \pm 3.4]$ spurious pairs. Dividing these numbers by the numbers of photo$z$ pair candidates, the predicted SPF in the four photo- $z$ bins are $0.07 \pm 0.03,0.08 \pm 0.03,0.10 \pm 0.03$, and $0.09 \pm 0.03$, respectively. 

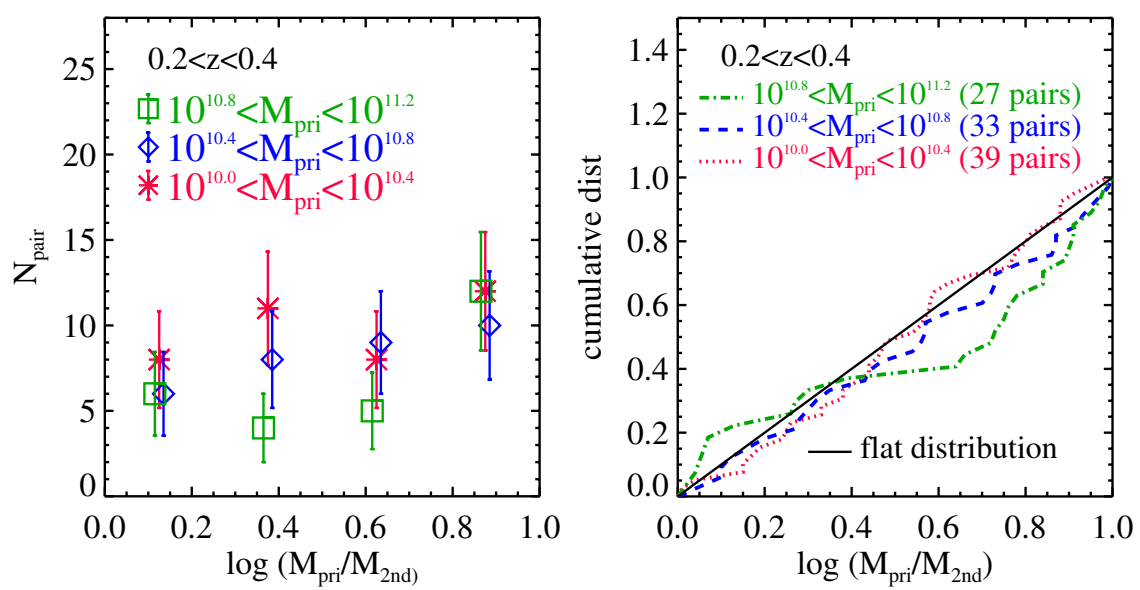

Figure 18. Left: differential distributions of $\log (\mu)\left(\mu=M_{\text {pri }} / M_{2 \text { nd }}\right)$ of close pairs $\left(5 h^{-1} \leqslant r_{\text {proj }} \leqslant 20 h^{-1} \mathrm{kpc}\right)$ in three mass bins. Derived using pair candidates selected in a volume-limited sample of galaxies with redshift in the range of $0.2 \leqslant z \leqslant 0.4$. Right: normalized cumulative distributions of $\log (\mu)\left(\mu=M_{\text {pri }} / M_{2 \mathrm{nd}}\right)$ of the same close pairs.

(A color version of this figure is available in the online journal.)

\section{APPENDIX C}

\section{DISTRIBUTION OF LINE-OF-SIGHT VELOCITY DIFFERENCE OF MAJOR-MERGER PAIRS}

Local major-merger pairs in Domingue et al. (2009) were selected using nearly identical selection criteria as the CPAIR sample (cf. Section 4) except that spec-z's were used and they include all pairs (isolated or in groups/clusters) with $\Delta v_{\text {spec }} \leqslant 1000 \mathrm{~km} \mathrm{~s}^{-1}$. Here we exploit these data to determine the cumulative distribution of $\Delta v$ and, in particular, the fraction of pairs with $500 \mathrm{~km} \mathrm{~s}^{-1}<\Delta v \leqslant 1000 \mathrm{~km} \mathrm{~s}^{-1}$. We assume that all pairs with $\Delta v>1000 \mathrm{~km} \mathrm{~s}^{-1}$ are spurious. In the sample of Domingue et al. (2009), which is $0.82 \%$ complete for the spec- $z, 135$ pairs have measured $v_{\text {spec }}$ for both components and $v_{\text {spec }}>2000 \mathrm{~km} \mathrm{~s}^{-1}$ (excluding pairs in the local supercluster). The cumulative distribution of $\Delta v$ of these pairs is plotted in Figure 16. From this distribution, we found that the fraction of pairs with $\Delta v>500 \mathrm{~km} \mathrm{~s}^{-1}$ is $9.6 \%$ with a random error of $2.5 \%$ (binomial statistics). It should be pointed out that the $\Delta v$ distribution is sensitive to the environment and to the pair separation (Ellison et al. 2010). Therefore, caution should be taken when applying the result here to other pair samples.

\section{APPENDIX D}

\section{INCOMPLETENESS AND SPURIOUS PAIRS FRACTION-A COMPARISON WITH SPEC- $z$ PAIRS}

We made a comparison between pairs in the CPAIR sample and a sample of spec- $z$ pairs (ZPAIR sample) selected from the zCOSMOS survey (Lilly et al. 2007). In principle, this comparison applies only to galaxies brighter than $i_{\mathrm{AB}}=22.5$, the magnitude limit of zCOSMOS. However, most of galaxies in photo- $z$ pairs are brighter than $i_{\mathrm{AB}}=22.5$ (Figure 15). Images of the 16 pairs in the ZPAIR sample, listed in Table 6 , are shown in Figure 17. Among them are all CPAIRs (14) where both components have spec- $z$ 's. They comprise a very small fraction of total CPAIR sample because only $19.5 \%$ of galaxies of $i_{\mathrm{AB}}<22.5$ in the parent sample have spec- $z$. All but two of the 14 pairs have the velocity difference $\Delta v_{\text {spec }}<500 \mathrm{~km} \mathrm{~s}^{-1}$. This results in an empirical estimate for the SPF of $2 / 14=0.14$, or a reliability of 0.86 , with a binomial uncertainty of \pm 0.12 .
Table 6 also includes two pairs (ZPAIR-03 and ZPAIR-07) that were missed by CPAIR sample, both having $\Delta z_{\text {phot }} /(1+$ $\left.z_{\text {phot }}\right)>0.03$ but $\Delta v_{\text {spec }}<500 \mathrm{~km} \mathrm{~s}^{-1}$. As shown in Figure 17, the primary of ZPAIR-03 is a close pair itself, and its large $z_{\text {phot }}$ error (Table 6) is likely due to confusion in the photometric data. ZPAIR-07, having $\Delta z_{\text {phot }} /\left(1+z_{\text {phot }}\right)=0.031$, barely missed the cut for the photo- $z$ pairs. Adding the 12 genuine CPAIRs, there are 14 pairs with $\Delta v<500 \mathrm{~km} \mathrm{~s}^{-1}$ in the ZPAIR sample. This results in a completeness for the CPAIR sample of $12 / 14=0.86$, with a statistical error 0.12 .

\section{APPENDIX E}

\section{DISTRIBUTION OF PRIMARY-TO-SECONDARY MASS RATIO OF CLOSE PAIRS}

In the parent sample of CPAIR, galaxies in the photo- $z$ bin of $0.2 \leqslant z_{\text {phot }} \leqslant 0.4$ form a volume-limited sample above the stellar mass limit $M_{\text {star }}=10^{9.0} M_{\odot}$. Using this volume-limited sample and applying the same pair selection criteria in Section 2 except for expanding the mass ratio limit to $\log \left(\mu_{\max }\right)=1.0$, where $\mu=M_{\text {pri }} / M_{2 \text { nd }}$, we selected pair candidates (including both major and minor mergers) in three mass bins: $10.0<$ $\log \left(M_{\text {pri }} / M_{\odot}\right) \leqslant 10.4,10.4<\log \left(M_{\text {pri }} / M_{\odot}\right) \leqslant 10.8$, and $10.8<\log \left(M_{\text {pri }} / M_{\odot}\right) \leqslant 11.2$. Assuming that completeness and reliability corrections for these pair candidates do not depend on the mass ratio $\mu$, we calculated the differential and cumulative $\log (\mu)$ distributions of these close pairs $\left(5 h^{-1} \mathrm{kpc} \leqslant r_{\text {proj }} \leqslant\right.$ $\left.20 h^{-1} \mathrm{kpc}\right)$. The results are plotted in Figure 18. It shows that the flat distribution, i.e., $d f_{\text {pair }} / d \log (\mu)=$ constant, is a reasonably good approximation. It is worth noting that our result is different from that of Ellison et al. (2010), who found a mass ratio distribution for SDSS pairs that is tilted toward low $\mu$ pairs (i.e., major mergers). However, their result is affected significantly by the "missing secondary" bias, causing severe incompleteness of the $\mu \sim 10$ minor mergers in their sample.

\section{REFERENCES}

Bamford, S. P., Nichol, R. C., Baldry, I. K., et al. 2009, MNRAS, 393, 1324 Barnes, J. E. 1988, ApJ, 331, 699

Bell, E. F., Papovich, C., Wolf, C., et al. 2005, ApJ, 625, 23

Bell, E. F., Phleps, S., Somerville, R. S., et al. 2006, ApJ, 652, 270 
Bell, E. F., Zheng, X. Z., Papovich, C., et al. 2007, ApJ, 663, 834 Bensen, A. J., Bower, R. G., Frenk, C. S., et al. 2003, ApJ, 599, 38 Bertin, E., \& Arnouts, S. 1996, A\&AS, 117, 393

Binney, J., \& Tremaine, S. 1987, in Galactic Dynamics, ed. J. Binney \& S. Tremaine (Princeton, NJ: Princeton Univ. Press), 747

Bridge, C. R., Appleton, P. N., Conselice, C. J., et al. 2007, ApJ, 659, 931

Brinchmann, J., Abraham, R., Shade, D., et al. 1998, ApJ, 499, 112

Bundy, K., Fukugita, M., Ellis, R. S., et al. 2009, ApJ, 697, 1369

Bundy, K., Scarlata, C., Carollo, C. M., et al. 2010, ApJ, 719, 1969

Capak, P., Abraham, R. G., Ellis, R. S., et al. 2007, ApJS, 172, 284

Carlberg, R. G., Cohen, J. G., Patton, D. R., et al. 2000, ApJ, 532, L1

Conselice, C. J. 2006, ApJ, 638, 686

Conselice, C. J., Bershady, M. A., Dickinson, M., \& Papovich, C. 2003, AJ, 126,1183

Conselice, C. J., Yang, C. C., \& Bluck, A. F. L. 2009, MNRAS, 394, 1956

Daddi, E., Elbaz, D., Walter, F., et al. 2010, ApJ, 714, L118

Dasyra, K. M., Tacconi, L. J., Davies, R. I., et al. 2006, ApJ, 638, 745

de Propris, R., Conselice, C. J., Liske, J., et al. 2007, ApJ, 666, 212

de Ravel, L., Le Fèvre, O., Tresse, L., et al. 2009, A\&A, 498, 379

Domingue, D. L., Xu, C. K., Jarrett, T. H., \& Cheng, Y.-H. 2009, ApJ, 695, 1559

Drory, N., \& Alvares, M. 2008, ApJ, 680, 41

Drory, N., Bundy, K., Leauthaud, A., et al. 2009, ApJ, 707, 1595

Ellison, S. L., Patton, D. R., Simard, L., et al. 2010, MNRAS, 407, 1514

Faber, S. M., Willmer, C. N. A., Wolf, C., et al. 2007, ApJ, 665, 265

Flores, H., Hammer, F., Thuan, T. X., et al. 1999, ApJ, 517, 148

Foucaud, S., Conselice, C. J., Hartley, W. G., et al. 2010, MNRAS, 406, 147

Genzel, R., Tacconi, L. J., Rigopoulou, D., Lutz, D., \& Tecza, M. 2001, ApJ, 563,527

Hammer, F., Flores, H., Elbaz, D., et al. 2005, A\&A, 430, 115

Hibbard, J. E., \& van Gorkom, J. H. 1996, AJ, 111, 655

Hibbard, J. E., \& Yun, M. S. 1999, ApJ, 522, 93

Hopkins, P. F., Bundy, K., Croton, D., et al. 2010a, ApJ, 715, 202

Hopkins, P. F., Cox, T. J., Kere, D., \& Hernquist, L. 2008, ApJS, 75, 390

Hopkins, P. F., Cox, T. J., Younger, J. D., \& Hernquist, L. 2009, ApJ, 691, 1168

Hopkins, P. F., Croton, D., Bundy, K., et al. 2010b, ApJ, 724, 915

Huertas-Company, M., Aguerri, J. A. L., Tresse, L., et al. 2010, A\&A, 515, 3

Ilbert, O., Capak, P., Salvato, M., et al. 2009, ApJ, 690, 1236

Ilbert, O., Salvato, M., Le Floc'h, E., et al. 2010, ApJ, 709, 644

Jiang, C. Y., Jing, Y. P., Faltenbacher, A., Lin, W. P., \& Li, C. 2008, ApJ, 675, 1095

Jogee, S., Miller, S. H., Penner, K., et al. 2009, ApJ, 697, 1971

Kampczyk, P., Lilly, S. J., Carollo, C. M., et al. 2007, ApJS, 172, 329

Kannappan, S. J., Guie, J. M., \& Baker, A. J. 2009, AJ, 138, 579

Kartaltepe, J. S., Sanders, D. B., Le Floc'h, E., et al. 2010, ApJ, 721, 98

Kartaltepe, J. S., Sanders, D. B., Scoville, N. Z., et al. 2007, ApJS, 172, 320

Kauffmann, G., Heckman, T. M., White, S. D. M., et al. 2003, MNRAS, 341, 33

Kauffmann, G., White, S. D. M., \& Guiderdoni, B. 1993, MNRAS, 264, 201

Kennicutt, R. C., Keel, W., van der Hulst, J., et al. 1987, AJ, 93, 1001

Khochfar, S., \& Burkert, A. 2005, MNRAS, 359, 1379
Kitzbichler, M. G., \& White, S. D. M. 2008, MNRAS, 391, 1488

Koekemoer, A. M., Aussel, H., Calzetti, D., et al. 2007, ApJS, 172, 196

Kormendy, J., \& Kennicutt, R. C. 2004, ARA\&A, 42, 603

Lacey, C., \& Cole, S. 1993, MNRAS, 262, 627

Larson, R. B., \& Tinsley, B. M. 1978, ApJ, 219, 46

Leauthaud, A., Finoguenov, A., Kneib, J.-P., et al. 2010, ApJ, 709, 97

Leauthaud, A., Massey, R., Kneib, J.-P., et al. 2007, ApJS, 172, 219

LeFévre, O., Abraham, R., \& Lilly, S. J. 2000, MNRAS, 311, 565

Lilly, S. J., LeFévre, O., Renzini, A., et al. 2007, ApJS, 172, 70

Lin, L., Koo, D. C., Wilmer, C. N. A., et al. 2004, ApJ, 617, L9

Lin, L., Patton, D. R., \& Koo, D. C. 2008, ApJ, 681, 232

Lotz, J. M., Davis, M., Faber, S. M., et al. 2008, ApJ, 672, 177

Lotz, J. M., Jonsson, P., Cox, T. J., \& Primack, J. R. 2010, MNRAS, 404, 575

Man, A. W. S., Troft, S., Zirm, A. W., Wuyts, S., \& van der Well, A. 2011, arXiv: 1109.2985

Martig, M., Bournaud, F., Teyssier, R., \& Dekel, A. 2009, ApJ, 707, 250

Melbourne, J., Koo, D. C., \& Flóch, E. L. 2005, ApJ, 632, L65

Meneux, B., Guzzo, L., Garilli, B., et al. 2008, A\&A, 478, 299

Patton, D. R., \& Atfield, J. E. 2008, ApJ, 685, 235

Patton, D. R., Carlberg, R. G., Marzke, R. O., et al. 2000, ApJ, 536, 153

Patton, D. R., Pritchet, C. J., \& Carlberg, R. G. 2002, ApJ, 565, 208

Peebles, P. J. E. 1980, in The Large-Scale Structure of the Universe, ed. P. J. E. Peebles (Princeton, NJ: Princeton Univ. Press), 435

Peng, Y., Lilly, S. J., Kovač, K., et al. 2010, ApJ, 721, 193

Rawat, A., Hammer, F., Kembhavi, A. K., \& Flores, H. 2008, ApJ, 681, 1089

Robaina, A. R., Bell, E. F., van der Well, A., et al. 2010, ApJ, 719, 844

Sanders, D. B., \& Mirabel, I. F. 1996, ARA\&A, 34, 749

Sanders, D. B., Salvato, M., Aussel, H., et al. 2007, ApJS, 172, 86

Sanders, D. B., Soifer, B. T., Elias, J. H., et al. 1988, ApJ, 325, 74

Schweizer, F. 1982, ApJ, 252, 455

Schweizer, F. 1996, in Saas-Fee Advanced Course, Vol. 26, Galaxies: Interactions and Induced Star Formation, ed. D. Friedli, L. Martinet, \& D. Pfenniger (Berlin: Springer), 105

Scoville, N. Z., Aussel, H., Brusa, M., et al. 2007, ApJS, 172, 1

Somerville, R. S., Hopkins, P. F., Cox, T. J., Robertson, B. E., \& Hernquist, L. 2008, MNRAS, 391, 481

Somerville, R. S., Lee, K., Ferguson, H. C., et al. 2004, ApJ, 600, L171

Stewart, K. R., Bullock, J. S., Barton, E. J., \& Wechsler, R. H. 2009, ApJ, 702, 1005

Toomre, A. 1978, in The Evolution of Galaxies and Stellar Populations, ed. B. M. Tinsley, R. B. Larson, \& D. Campbell (New Haven, CT: Yale Univ. Press), 401

Toomre, A., \& Toomre, M. 1972, ApJ, 178, 623

van den Bosch, F. C., Aquino, D., Yang, X., et al. 2008, MNRAS, 387, 79

Wang, Z., Fazio, G. G., Ashby, M. L. N., et al. 2004, ApJS, 154, 193

Whitmore, B. C., \& Schweizer, F. 1995, AJ, 109, 960

Xu, C. K., Domingue, D., Cheng, Y., et al. 2010, ApJ, 713, 330

Xu, C., Gao, Y., Mazzarella, J., et al. 2000, ApJ, 541, 644

Xu, C., \& Sulentic, J. W. 1991, ApJ, 374, 407

Xu, C. K., Sun, Y. C., \& He, X. T. 2004, ApJ, 603, L73

Zehavi, I., Zheng, Z., \& Weinberg, D. 2005, ApJ, 630, 1

Zheng, X. Z., Hammer, F., Flores, H., et al. 2004, A\&A, 421, 847 